\title{
CENTERED HARDY-LITTLEWOOD MAXIMAL FUNCTIONS ON HEISENBERG TYPE GROUPS
}

\author{
HONG-QUAN LI AND BIN QIAN
}

\begin{abstract}
In this paper, by establishing uniform lower bounds for the Poisson kernel and $(-\Delta)^{-\frac{1}{2}}$ on the Heisenberg type group $\mathbb{H}(2 n, m)$ with $m \geq 2$, which follow from the various properties of Bessel functions and Legendre functions, we prove that there exists a constant $A>0$ such that, for all $f \in L^{1}(\mathbb{H}(2 n, m))$ and all $n, m \in \mathbb{N}^{*}$ satisfying $4 \leq m^{2} \ll \log n$, we have $\left\|M_{K} f\right\|_{L^{1, \infty}} \leq A n\|f\|_{1}$, where $M_{K}$ denotes the centered Hardy-Littlewood maximal function defined by the Korányi norm. For the centered Hardy-Littlewood maximal function $M_{C C}$ defined by the Carnot-Carathédory distance, we prove $\left\|M_{C C} f\right\|_{L^{1, \infty}} \leq$ $A(m) n\|f\|_{1}$ holds for some constant $A(m)$ independent of $n$.
\end{abstract}

\section{INTRODUCTION}

Consider the standard centered Hardy-Littlewood maximal function, $M_{\mathbb{R}^{n}}$, in $\mathbb{R}^{n}\left(n \in \mathbb{N}^{*}\right)$, i.e.

$$
M_{\mathbb{R}^{n}} f(x)=\sup _{r>0} \frac{1}{\left|B_{\mathbb{R}^{n}}(x, r)\right|} \int_{B_{\mathbb{R}^{n}(x, r)}}|f(y)| d y, \quad x \in \mathbb{R}^{n}, f \in L_{l o c}^{1}\left(\mathbb{R}^{n}\right),
$$

where $d y$ is the Lebesgue measure and $\left|B_{\mathbb{R}^{n}}(x, r)\right|$ is the volume of the euclidean ball with the center $x \in \mathbb{R}^{n}$ and the radius $r>0$.

By the tripling property of the volume, i.e.

$$
\left|B_{\mathbb{R}^{n}}(x, 3 r)\right| \leq 3^{n}\left|B_{\mathbb{R}^{n}}(x, r)\right|, \quad \forall x \in \mathbb{R}^{n}, r>0,
$$

one deduces from the Vitali covering lemma that $M_{\mathbb{R}^{n}}$ satisfies the weak type $(1,1)$ bounds with

$$
\left\|M_{\mathbb{R}^{n}}\right\|_{L^{1} \longrightarrow L^{1, \infty}} \leq 3^{n} .
$$

However, applying the Hopf-Dunford-Schwartz maximal ergodic theorem, Stein and Strömberg obtained in [20] via the heat kernel that there exists a constant $A>0$ such that

$$
\left\|M_{\mathbb{R}^{n}}\right\|_{L^{1} \longrightarrow L^{1, \infty}} \leq A \phi(n), \forall n, \quad \text { with } \phi(n)=n .
$$

In the setting of the Heisenberg groups, $\mathbb{H}(2 n, 1)$, the estimate of type (1.1) has been obtained for the centered Hardy-Littlewood maximal function defined by the Carnot-Carathéodory distance or by the Korányi norm. The proof is based on a uniform lower estimate of the Poisson kernel (the integral kernel of the Poisson semigroup; there is no relation with the one in [4]); see [10] for details.

Received by the editors February 10, 2012.

2010 Mathematics Subject Classification. Primary 42B25, 43A80 .

(C) 2013 American Mathematical Society Reverts to public domain 28 years from publication 
For the maximal function $M_{G}$ associated to the Carnot-Carathéodory distance or the pseudo-distance induced by the fundamental solution to the Grushin operator

$$
\Delta_{G}=\sum_{i=1}^{n} \frac{\partial^{2}}{\partial x_{i}^{2}}+\left(\sum_{i=1}^{n} x_{i}^{2}\right) \frac{\partial^{2}}{\partial u^{2}},
$$

the first named author has obtained estimate (1.1) for $M_{G}$ in [12].

As mentioned in [12, the above three results can be explained roughly by an estimate of the following type:

$$
\inf _{n \geq 3, h>0, g \neq g^{\prime} \in B(g, h)} \phi(n) \frac{n}{h^{2}}|B(g, h)|(-\Delta)^{-1}\left(g, g^{\prime}\right)>0, \quad \text { with } \phi(n)=n,
$$

in the euclidean spaces, Heisenberg groups and for the Grushin operators. Furthermore, we believe that there is a close relation between the estimate of type (1.1) (of course, the volume of the ball and the dimension play their roles) and the Green function. In fact, the work 12 is motivated by the estimate (1.2). Also, the results in [20, [10] and 12] can be explained by an estimate of the following type:

$$
\inf _{n \geq 3, h>0, g \neq g^{\prime} \in B(g, h)} \phi(n) \frac{\sqrt{n}}{h}|B(g, h)|(-\Delta)^{-\frac{1}{2}}\left(g, g^{\prime}\right)>0 .
$$

Following the above idea and applying various properties of the real hyperbolic spaces of dimension $n(n \geq 2)$ which are measured metric spaces of exponential volume growth, Li and Lohoué showed in 15 that (1.1) holds with $\phi(n)=n \ln n$ in this case. A similar method works for the harmonic $A N$ groups. For other $O(n \ln n)$ results, see [20] and [17].

We remark that up to a universal constant, the two terms $\frac{n}{h^{2}}$ and $\frac{\sqrt{n}}{h}$, which can be found in (1.2) and (1.3) respectively, are optimal. Note that it suffices to take $h=1$ in the above three models, i.e. $\mathbb{R}^{n}, \mathbb{H}(2 n, 1)$ and $\Delta_{G}$ (thanks to the dilation structure); see [10] and [12] for details.

The purpose of this paper is to use this idea to show (1.1) holds on the Heisenberg type groups $\mathbb{H}(2 n, m)$. We suppose $m \geq 2$ in what follows, rather than the case $m=$ 1 which has been treated in [10]. We will see it is necessary to distinguish the case of $m=1$ from the one of $m \geq 2$. The proof for $m=1$ is very natural, however the one for $m \geq 2$ is much more technical (it is based on the various properties of Bessel functions and Legendre functions), and the technique used for $m \geq 2$ does not work for $m=1$ because of the properties of Legendre functions. The method here follows the ones in [10,12, but it is not enough to get the desired result only by using the Poisson kernel or $(-\Delta)^{-\frac{1}{2}}\left(g, g^{\prime}\right)$ and $(-\Delta)^{-\frac{1}{2}} \exp \left(-\omega \frac{d_{K}\left(g, g^{\prime}\right)}{\sqrt{n}} \sqrt{-\Delta}\right)\left(g, g^{\prime}\right)$ alone. Here we must take both of them into consideration. To this end, we should make good use of various properties of Bessel functions and Legendre functions. Note that using the same method in [10, Zhao and Song in 25] obtained (1.1) in the special case $\mathbb{H}(2 n, 3)$ for the centered Hardy-Littlewood maximal functions defined by the Korányi norm.

However, (1.1) has been obtained in the setting of $S^{n-1}(n \geq 2)$, the unit sphere of dimension $n-1$ (i.e. the $n-1$ dimensional, simply connected Riemannian manifold of constant sectional curvature 1); see [9] and [14. In this case, we can't use (1.2) or (1.3) to explain this bound since $\left(-\Delta_{S^{n-1}}\right)^{-1}$ and $\left(-\Delta_{S^{n-1}}\right)^{-\frac{1}{2}}$ do not exist.

Let us first introduce the concept of the Heisenberg type groups $\mathbb{H}(2 n, m)$ and centered Hardy-Littlewood maximal functions. 


\subsection{Heisenberg-type groups and centered Hardy-Littlewood maximal}

functions. Recall that a Heisenberg type group (for short, H-type) can be considered as $\mathbb{H}(2 n, m)=\mathbb{R}^{2 n} \times \mathbb{R}^{m}\left(m, n \in \mathbb{N}^{*}\right)$ with the group law (see e.g. Theorem A.2, p. 199 in [3], and one can refer to $[8$ for the original definition)

$$
(x, t) \cdot\left(x^{\prime}, t^{\prime}\right)=\left(x+x^{\prime}, t+t^{\prime}+2^{-1}\left\langle x, U x^{\prime}\right\rangle\right),
$$

with $x=\left(x_{1}, \ldots, x_{2 n}\right) \in \mathbb{R}^{2 n}, t=\left(t_{1}, \ldots, t_{m}\right) \in \mathbb{R}^{m}$ and

$$
\left\langle x, U x^{\prime}\right\rangle=\left(\left\langle x, U^{(1)} x^{\prime}\right\rangle, \ldots,\left\langle x, U^{(m)} x^{\prime}\right\rangle\right) \in \mathbb{R}^{m},
$$

where the matrices $U^{(1)}, \cdots, U^{(m)}$ satisfy the following two conditions:

1. $U^{(j)}$ is a $2 n \times 2 n$ anti-symmetric and orthogonal matrix, for all $j=1,2, \cdots, m$;

2. $U^{(i)} U^{(j)}+U^{(j)} U^{(i)}=0$, for all $i, j \in\{1, \cdots, m\}$ with $i \neq j$.

Let $U^{(j)}=\left(U_{k, l}^{(j)}\right)_{k, l \leq 2 n}(1 \leq j \leq m)$. The canonical sub-Laplacian on $\mathbb{H}(2 n, m)$ can be written as $\Delta=\sum_{l=1}^{2 n} X_{l}^{2}$, where $X_{l}(1 \leq l \leq 2 n)$ are the left invariant vector fields on $\mathbb{H}(2 n, m)$, defined by

$$
X_{l}=\frac{\partial}{\partial x_{l}}+\frac{1}{2} \sum_{j=1}^{m}\left(\sum_{k=1}^{2 n} x_{k} U_{k, l}^{(j)}\right) \frac{\partial}{\partial t_{j}} .
$$

Notice that $\mathbb{H}(2 n, 1)$ is the exact Heisenberg group of dimension $2 n+1$. Recall that, as in [8], $(2 n, m)$ satisfies the following condition: $2 n=(2 a+1) 2^{4 p+q}$ with $a, p \in \mathbb{N}^{*}$ and $0 \leq q<3$, and it implies

$$
m<\rho(2 n)=8 p+2^{q} .
$$

In particular, the following estimate will be used in this paper:

$$
m \leq 2 \log _{2}(2 n) .
$$

Let $o=(0,0)$ denote the identity of $\mathbb{H}(2 n, m)$ and $g=(x, t) \in \mathbb{R}^{2 n} \times \mathbb{R}^{m}$ denote a point of $\mathbb{H}(2 n, m)$. We use the following notation through the whole paper:

$$
|x|^{2}=\sum_{k=1}^{2 n} x_{k}^{2}, \quad|t|^{2}=\sum_{j=1}^{m} t_{j}^{2} \text { and } t \cdot \lambda=\sum_{j=1}^{m} t_{j} \lambda_{j} \text { for } \lambda \in \mathbb{R}^{m} .
$$

Recall that the Haar measure $d g$ on $\mathbb{H}(2 n, m)$ is the Lebesgue measure.

There are two standard distances on $\mathbb{H}(2 n, m)$ : one is the Carnot-Carathéodory distance $d_{C C}$ (associated to $\left\{X_{1}, \cdots, X_{2 n}\right\}$; see [23]), and the other one is defined by the Korányi norm $d_{K}$, which is associated to the fundamental solution of $\Delta$ (i.e. the Green function; see for example [8]). Moreover, one has

$$
d_{C C}\left(g g_{1}, g g_{2}\right)=d_{C C}\left(g_{1}, g_{2}\right), d_{K}\left(g g_{1}, g g_{2}\right)=d_{K}\left(g_{1}, g_{2}\right), \forall g, g_{1}, g_{2} \in \mathbb{H}(2 n, m) \text {. }
$$

By convention, denote $d_{K}(g)=d_{K}(g, o)$ and $d_{C C}(g)=d_{C C}(g, o)$. Recall that (see for example [8])

$$
d_{K}(x, t)=\left(|x|^{4}+16|t|^{2}\right)^{\frac{1}{4}}, \quad \forall(x, t) \in \mathbb{H}(2 n, m)=\mathbb{R}^{2 n} \times \mathbb{R}^{m} .
$$

Denote $\left.\mu(\varphi)=(2 \varphi-\sin 2 \varphi) /\left(2 \sin ^{2} \varphi\right):\right]-\pi, \pi\left[\longrightarrow \mathbb{R}\right.$ and let $\mu^{-1}$ be the inverse function. One has $d_{C C}^{2}(0, t)=4 \pi|t|$ and for $x \neq 0$ (see [19] or [22], pp. 90-91),

$$
d_{C C}^{2}(x, t)=(\theta / \sin \theta)^{2}|x|^{2} \text { with } \theta=\mu^{-1}\left(4|t| /|x|^{2}\right) .
$$

In what follows, one denotes $B_{K}(g, r)(g \in \mathbb{H}(2 n, m), r>0)$ the open ball with the center $g$ and the radius $r$ induced by the Korányi norm $d_{K}$, and $B_{C C}(g, r)$ 
induced by the Carnot-Carathéodory distance $d_{C C}$. For a measurable set $E$, let $|E|$ denote the volume and $\chi_{E}$ the characteristic function. For $f \in L_{l o c}^{1}(\mathbb{H}(2 n, m))$, one can define two centered Hardy-Littlewood maximal functions respectively by

$$
\begin{gathered}
M_{K} f(g)=\sup _{r>0}\left|B_{K}(g, r)\right|^{-1} \int_{B_{K}(g, r)}\left|f\left(g^{\prime}\right)\right| d g^{\prime}, \quad \forall g \in \mathbb{H}(2 n, m), \\
M_{C C} f(g)=\sup _{r>0}\left|B_{C C}(g, r)\right|^{-1} \int_{B_{C C}(g, r)}\left|f\left(g^{\prime}\right)\right| d g^{\prime}, \quad \forall g \in \mathbb{H}(2 n, m) .
\end{gathered}
$$

The main result is the following

Theorem 1.1. There exists a constant $A>0$ such that for all $n, m \in \mathbb{N}^{*}$ satisfying $m^{2} \ll \log n$, we have

$$
\left\|M_{K} f\right\|_{L^{1, \infty}} \leq A n\|f\|_{1}, \quad \forall f \in L^{1}(\mathbb{H}(2 n, m)) .
$$

Furthermore, for any fixed $m \in \mathbb{N}^{*}$, we have

$$
\left\|M_{C C} f\right\|_{L^{1, \infty}} \leq L(m) n\|f\|_{1}, \quad \forall n, \forall f \in L^{1}(\mathbb{H}(2 n, m)),
$$

for some constant $L(m)>0$ independent of $n$.

Remark 1.2. (1) Notice that (1.2) holds for all $\mathbb{H}(2 n, m)$ (see section 3 below). We believe it is most possible to show (1.8) holds for all $\mathbb{H}(2 n, m)$. By applying more properties of Bessel functions and Legrendre functions, we may prove it.

(2) Moreover, by a certain property of Beta function, we could get an upper bound for $L(m)$ in (1.9).

(3) From the result obtained by Naor and Tao [17] or by [20, we have

$$
\|M f\|_{L^{1} \rightarrow L^{1, \infty}} \leq L(2 n+2 m) \ln (2 n+2 m), \forall(2 n, m)
$$

holds with $M=M_{K}$ or $M=M_{C C}$ for some positive constant $L$. For $m$ fixed, as $n \rightarrow \infty,(1.8)$ and (1.9) are better than (1.10). When $m \rightarrow \infty$, for $M=M_{C C}$, (1.9) is not always better.

(4) Observe that there exists a bound of type $\lim _{n \longrightarrow+\infty}\left\|M_{C u b e}\right\|_{L^{1} \longrightarrow L^{1, \infty}}=$ $+\infty$ for the centered maximal functions associated to cubes in $\mathbb{R}^{n}$; see [1] for details.

\section{OUTLINE OF THE PROOF}

The main idea of the proof has been pointed out in [12]. More precisely, by certain results in 10, we need only to prove (1.8); by the recursion formula obtained in [11, it is enough to consider the case of $m \geq 3$ odd. To this end, we divide the proof into two cases: in the first case, we get the uniform lower bound for the Poisson kernel; in the other case, we obtain good estimations of $(-\Delta)^{-\frac{1}{2}}(g)$ and $(-\Delta)^{-\frac{1}{2}} \exp \left(-\omega \frac{d_{K}(g)}{\sqrt{n}} \sqrt{-\Delta}\right)(g)$.

This paper is organized as follows: some properties of Bessel functions and Legendre functions are presented in section 2, In section 3 we review the heat kernel, the Poisson kernel and Green function on $\mathbb{H}(2 n, m)$. In section 4, we prove Theorem 1.1 for $m \geq 3$ odd and $d_{K}$ metric, and Theorem 1.1 for $m \geq 2$ even and $d_{K}$ metric is proved in section 5. In section 6, we prove Theorem 1.1 for $d_{C C}$ metric. 


\section{NOTATION}

We shall use $C, C^{\prime}, A, A^{\prime}$, etc. to denote absolute positive constants whose value may differ at each occurrence.

For two functions $f$ and $g$, we denote $f=O(g)$ if there exists a constant $c>0$ such that $|f| \leq c|g|, f=o(g)$ if $\lim \frac{f}{g}=0$, and $f \sim g$ if there exists an $A>1$ such that $A^{-1} f \leq g \leq A f$.

\section{Review of Bessel functions And Legendre Functions}

In what follows, we denote $J_{\nu}$ the Bessel function, for $\Re \nu>-\frac{1}{2}$ and $-\pi<$ $\arg z<\pi$, defined by (see for example [16], p. 65 and p. 79)

$$
J_{\nu}(z)=\sum_{k=0}^{+\infty} \frac{(-1)^{k}(z / 2)^{\nu+2 k}}{k ! \Gamma(\nu+k+1)}=\frac{2}{\sqrt{\pi} \Gamma\left(\frac{1}{2}+\nu\right)}\left(\frac{z}{2}\right)^{\nu} \int_{0}^{1}\left(1-h^{2}\right)^{\nu-\frac{1}{2}} \cos (z h) d h .
$$

In particular, we have the following estimate which will be used repeatedly:

$$
(z / 2)^{-\nu}\left|J_{\nu}(z)\right| \leq \frac{1}{\Gamma(\nu+1)}, \quad z>0 .
$$

Recall that (see for example $\S 3.1 .1$ in [16], p. 67 , and $\S 8.472$ in [6], p. 926 , or $\S 3.2$ in [24], p. 46)

$$
\frac{1}{z} \frac{d}{d z}\left(z^{-\nu} J_{\nu}(z)\right)=-z^{-\nu-1} J_{\nu+1}(z)
$$

In the case of $m \geq 3$ odd, $J_{\frac{m-2}{2}}(\lambda)$ is a simple function; see for example [16], p. 72 , or $\S 8.46$ in [6], pp. $924-925$, or [24], p. 53. More precisely,

$$
J_{\frac{m-2}{2}}(z)=T_{1}(z)+T_{2}(z)
$$

where

$$
\begin{aligned}
& T_{1}(z)=\frac{1}{\sqrt{2 \pi z}} e^{-i \frac{\pi}{2} \frac{m-1}{2}} e^{i z} \sum_{k=0}^{\frac{m-3}{2}} \frac{i^{k} \Gamma\left(\frac{m-1}{2}+k\right)}{k ! \Gamma\left(\frac{m-1}{2}-k\right)(2 z)^{k}}, \\
& T_{2}(z)=\frac{1}{\sqrt{2 \pi z}} e^{i \frac{\pi}{2} \frac{m-1}{2}} e^{-i z} \sum_{k=0}^{\frac{m-3}{2}} \frac{(-i)^{k} \Gamma\left(\frac{m-1}{2}+k\right)}{k ! \Gamma\left(\frac{m-1}{2}-k\right)(2 z)^{k}} .
\end{aligned}
$$

Recall that for $\Re(\mu+\nu)>-1$ and $\Re s>|\Im a|$, one has (see [16], p. 446)

$$
\int_{0}^{+\infty} \lambda^{\mu} J_{\nu}(a \lambda) e^{-s \lambda} d \lambda=\Gamma(\mu+\nu+1)\left(s^{2}+a^{2}\right)^{-\frac{1}{2}(\mu+1)} P_{\mu}^{-\nu}\left(\frac{s}{\sqrt{s^{2}+a^{2}}}\right),
$$

where $P_{\mu}^{-\nu}(r)(-1<r<1)$ is the Legendre function ("on the cut") with the parameters $\mu$ and $-\nu$.

When $n \in \mathbb{N}^{*}$ and $m \geq 2$ satisfies (1.4), one has for all $0<\theta \leq \frac{\pi}{2}$ (see [16], p. 188 and p. 203)

$$
\left|P_{n+\frac{m-1}{2}}^{-\frac{m-2}{2}}(\cos \theta)\right|<4 \frac{\Gamma\left(n+\frac{3}{2}\right)}{\Gamma\left(n+\frac{m-1}{2}+1\right)} \pi^{-\frac{1}{2}}\left(n+\frac{m-1}{2}\right)^{-\frac{1}{2}}(\sin \theta)^{-\frac{m-1}{2}} .
$$


Throughout this paper, we will adopt the following estimate (see for example [16], p. 12):

$$
\Gamma(s)=\sqrt{2 \pi} s^{-\frac{1}{2}}\left(\frac{s}{e}\right)^{s}\left[1+O\left(s^{-1}\right)\right], \quad s \gg 1 .
$$

\section{Review of the heat Kernel, Poisson Kernel AND GREen function on $\mathbb{H}(2 n, m)$}

Let $p_{h}=p_{h}^{(2 n, m)}(h>0)$ be the heat kernel (i.e. the kernel of $\left.e^{h \Delta}\right)$ on $\mathbb{H}(2 n, m)$, $P_{h}=P_{h}^{(2 n, m)}$ the Poisson kernel (i.e. the kernel of $e^{-h \sqrt{-\Delta}}$ ) and $G=G^{(2 n, m)}$ the Green function (i.e. the kernel of $\left.(-\Delta)^{-1}\right)$. By convention, one denotes $p_{h}(g)=$ $p_{h}(g, o), P_{h}(g)=P_{h}(g, o), P(g)=P_{1}(g)$ and $G(g)=G(g, o)$, where $o$ is the identity of $\mathbb{H}(2 n, m)$.

The heat kernel. We will need the following expression of $p_{h}^{(2 n, m)}(x, t)$, due to Randall ([18], p. 292):

$$
\begin{aligned}
p_{h}(x, t) & =2(4 \pi)^{-n-\frac{m}{2}} h^{-n-m} \\
& \times \int_{0}^{+\infty} r^{m-1} e^{-\frac{|x|^{2} r}{4 h} \operatorname{coth} r}\left(\frac{r}{\sinh r}\right)^{n}\left(\frac{r|t|}{2 h}\right)^{-\frac{m-2}{2}} J_{\frac{m-2}{2}}\left(\frac{r|t|}{h}\right) d r .
\end{aligned}
$$

In particular,

$$
p_{h}(x, t)=h^{-n-m} p(x / \sqrt{h}, t / h) .
$$

For fixed $m, n \in \mathbb{N}^{*}, w_{1}, w_{2} \geq 0$, denote

$$
p\left(n, m, w_{1}, w_{2}\right)=2(4 \pi)^{-n-\frac{m}{2}}
$$

$$
\times \int_{0}^{+\infty} r^{m-1} \exp \left(-\frac{w_{1} r}{4} \operatorname{coth} r\right)\left(\frac{r}{\sinh r}\right)^{n}\left(\frac{w_{2} r}{2}\right)^{-\frac{m-2}{2}} J_{\frac{m-2}{2}}\left(w_{2} r\right) d r .
$$

It follows that

$$
p(x, t)=p\left(n, m,|x|^{2},|t|\right), \quad \forall(x, t) \in \mathbb{H}(2 n, m) .
$$

By (2.3), we have (see also (1.12) in [1] )

$$
\frac{\partial}{\partial \omega_{2}} p\left(n, m, \omega_{1}, \omega_{2}\right)=-2 \pi \omega_{2} p\left(n, m+2, \omega_{1}, \omega_{2}\right) .
$$

Notice that, for $m, n \in \mathbb{N}^{*}$, for all $(x, t) \in \mathbb{H}(2 n, m)$ with $|t| \neq 0$, we have (see (1.13) in [11])

$$
p\left(n, m,|x|^{2},|t|\right)=2|t| \int_{1}^{\infty} \frac{w}{\sqrt{w^{2}-1}} p\left(n, m+1,|x|^{2}, w|t|\right) d w .
$$

The Poisson kernel. By the formula of subordination, i.e.

$$
e^{-\sqrt{-\Delta}}=\frac{1}{2 \sqrt{\pi}} \int_{0}^{+\infty} h^{-\frac{3}{2}} e^{-\frac{1}{4 h}} e^{h \Delta} d h,
$$

it follows from (3.1) and the Fubini theorem that

$$
P(x, t)=2(4 \pi)^{-n-\frac{m+1}{2}} \int_{0}^{+\infty} r^{m-1}\left(\frac{r}{\sinh r}\right)^{n} \mathrm{~T} d r,
$$


where

$$
\mathrm{T}=\int_{0}^{+\infty} h^{-n-m-\frac{3}{2}} e^{-\frac{1+|x|^{2} r \operatorname{coth} r}{4 h}}\left(\frac{r|t|}{2 h}\right)^{-\frac{m-2}{2}} J_{\frac{m-2}{2}}\left(\frac{r|t|}{h}\right) d h .
$$

For $\omega_{1} \geq 0, \omega_{2} \in \mathbb{R}^{+}$, denote

$$
P\left(n, m, \omega_{1}, \omega_{2}\right)=\frac{1}{2 \sqrt{\pi}} \int_{0}^{\infty} h^{-n-m-\frac{3}{2}} e^{-\frac{1}{4 h}} p\left(n, m, w_{1} / h, w_{2} / h\right) d h ;
$$

it follows that $P^{(2 n, m)}(x, t)=P\left(n, m,|x|^{2},|t|\right)$. Applying (3.5), one gets

$$
P\left(n, m,|x|^{2},|t|\right)=2|t| \int_{1}^{\infty} \frac{w}{\sqrt{w^{2}-1}} P\left(n, m+1,|x|^{2}, w|t|\right) d w .
$$

Let

$$
\mathrm{U}=\frac{1+|x|^{2} r \operatorname{coth} r}{4}, \quad \mathrm{~V}=\sqrt{\left(\frac{1+|x|^{2} r \operatorname{coth} r}{4}\right)^{2}+(|t| r)^{2}} .
$$

By (3.6) and (2.5), one can rewrite

$$
\begin{aligned}
& P\left(n, m,|x|^{2},|t|\right)=2(4 \pi)^{-n-\frac{m+1}{2}} \Gamma\left(n+m+\frac{1}{2}\right) \\
& \quad \times \int_{0}^{+\infty} r^{m-1}\left(\frac{r}{\sinh r}\right)^{n}\left(\frac{r|t|}{2}\right)^{-\frac{m-2}{2}} \mathrm{~V}^{-\left(n+\frac{m+1}{2}+1\right)} P_{n+\frac{m+1}{2}}^{-\frac{m-2}{2}}\left(\frac{\mathrm{U}}{\mathrm{V}}\right) d r .
\end{aligned}
$$

Let $Q=2 n+2 m$ be the homogenous dimension of $\mathbb{H}(2 n, m)$. We have the following time scaling property for the Poisson kernel:

$$
P_{h}(x, t)=h^{-Q} P\left(x / h, t / h^{2}\right), \quad \forall h>0,(x, t) \in \mathbb{R}^{2 n} \times \mathbb{R}^{m} .
$$

Note that for $m \geq 3$ odd, by (2.4) and (3.7), one has

$$
\begin{aligned}
& \mathrm{T}=\int_{0}^{+\infty} h^{-n-m-\frac{3}{2}} e^{-\frac{1+|x|^{2} r \operatorname{coth} r}{4 h}}\left(\frac{r|t|}{2 h}\right)^{-\frac{m-2}{2}}\left(T_{1}\left(\frac{r|t|}{h}\right)+T_{2}\left(\frac{r|t|}{h}\right)\right) d h \\
& =\frac{2^{\frac{m-3}{2}}}{\sqrt{\pi}(r|t|)^{\frac{m-1}{2}}} \sum_{k=0}^{\frac{m-3}{2}} \frac{\Gamma\left(\frac{m-1}{2}+k\right)}{k ! \Gamma\left(\frac{m-1}{2}-k\right) 2^{k}}(|t| r)^{-k} \\
& \times \int_{0}^{+\infty} h^{k-n-\frac{m}{2}-2} e^{-\frac{1+|x|^{2} r \operatorname{coth} r}{4 h}}\left[e^{\frac{i(|t| r)}{h}-i \frac{\pi}{2} \frac{m-1}{2}} i^{k}+e^{-\frac{i(|t| r)}{h}+i \frac{\pi}{2} \frac{m-1}{2}}(-i)^{k}\right] d h \\
& =\frac{2^{\frac{m-3}{2}}}{\sqrt{\pi}(r|t|)^{\frac{m-1}{2}}} \sum_{k=0}^{\frac{m-3}{2}} \frac{\Gamma\left(\frac{m-1}{2}+k\right)}{k ! \Gamma\left(\frac{m-1}{2}-k\right) 2^{k}}(|t| r)^{-k} \Gamma\left(n+\frac{m}{2}+1-k\right) \\
& \times\left\{e^{-i \frac{\pi}{2} \frac{m-1}{2}} i^{k}\left[\frac{1+|x|^{2} r \operatorname{coth} r}{4}-i(|t| r)\right]^{k-n-\frac{m}{2}-1}\right. \\
& \left.+e^{i \frac{\pi}{2} \frac{m-1}{2}}(-i)^{k}\left[\frac{1+|x|^{2} r \operatorname{coth} r}{4}+i(|t| r)\right]^{k-n-\frac{m}{2}-1}\right\} .
\end{aligned}
$$

Substituting into (3.6), it yields

$$
\begin{aligned}
P\left(n, m,|x|^{2},|t|\right) & =\frac{(4 \pi)^{-n-\frac{m+1}{2}} 2^{\frac{m-1}{2}} e^{-i \frac{\pi}{2} \frac{m-1}{2}}}{\sqrt{\pi}|t|^{\frac{m-1}{2}}} \sum_{k=0}^{\frac{m-3}{2}} \frac{\Gamma\left(\frac{m-1}{2}+k\right) i^{k} \Gamma\left(n+\frac{m}{2}+1-k\right)}{k ! \Gamma\left(\frac{m-1}{2}-k\right) 2^{k}|t|^{k}} \\
& \times \int_{\mathbb{R}} r^{\frac{m-1}{2}-k}\left(\frac{r}{\sinh r}\right)^{n}\left[\frac{1+|x|^{2} r \operatorname{coth} r}{4}-i(|t| r)\right]^{k-n-\frac{m}{2}-1} d r .
\end{aligned}
$$


Green function. For $(x, t) \in \mathbb{H}(2 n, m)$ with $|t| \neq 0$, the Green function $G(x, t)$ is the kernel of the operator $(-\Delta)^{-1}$ which can be expressed as

$$
\begin{aligned}
G(x, t) & =\int_{0}^{\infty} p_{h}(x, t) d h \\
& =2(4 \pi)^{-n-\frac{m}{2}} \int_{0}^{\infty} h^{-n-m} d h \\
& \times \int_{0}^{\infty} r^{m-1} \exp \left(-\frac{|x|^{2} r \operatorname{coth} r}{4 h}\right)\left(\frac{r}{\sinh r}\right)^{n}\left(\frac{r|t|}{2 h}\right)^{-\frac{m-2}{2}} J_{\frac{m-2}{2}}\left(\frac{r|t|}{h}\right) d r .
\end{aligned}
$$

Denote

$$
\mathfrak{G}\left(n, m, w_{1}, w_{2}\right)=\int_{0}^{\infty} h^{-n-m} p\left(n, m, w_{1} / h, w_{2} / h\right) d h, \omega_{1} \geq 0, \omega_{2} \in \mathbb{R}^{+},
$$

thus $G(x, t)=\mathfrak{G}\left(n, m,|x|^{2},|t|\right)$. For the Heisenberg group $\mathbb{H}(2 n, 1)$, i.e. $m=1$, we have (see [5, 7])

$$
\begin{aligned}
G^{(2 n, 1)}(x, t) & =\mathfrak{G}\left(n, 1,|x|^{2},|t|\right) \\
& =(2 \pi)^{-1}(4 \pi)^{-n} \Gamma(n) B\left(\frac{n}{2}, \frac{1}{2}\right)\left(\left(|x|^{2} / 4\right)^{2}+|t|^{2}\right)^{-\frac{n}{2}} .
\end{aligned}
$$

By (3.4), it follows that

$$
G^{(2 n, 2 k+1)}(x, t)=\mathfrak{G}\left(n, 2 k+1,|x|^{2},|t|\right)=\left(-\frac{1}{\pi} \frac{\partial}{\partial|t|^{2}}\right)^{k} \mathfrak{G}\left(n, 1,|x|^{2},|t|\right) .
$$

Combining this with (3.13), through direct computation, yields

$$
\begin{aligned}
G^{(2 n, 2 k+1)}(x, t) & =\mathfrak{G}\left(n, 2 k+1,|x|^{2},|t|\right) \\
& =\frac{1}{Q-2} 4^{m-\frac{1}{2}} \frac{\Gamma(n) \Gamma((Q+2) / 4)}{\pi^{n+\frac{m}{2}} \Gamma\left(\frac{n+1}{2}\right)} d_{K}^{2-Q}(x, t), m=2 k+1,
\end{aligned}
$$

which has been obtained by [7]; see also [8]. By (3.5), we have, for $(x, t) \in \mathbb{H}(2 n, 2 k)$,

$$
G^{(2 n, 2 k)}(x, t)=2|t| \int_{1}^{\infty} \frac{w}{\sqrt{w^{2}-1}} \mathfrak{G}\left(n, 2 k+1,|x|^{2}, w|t|\right) d w .
$$

Note that

$$
\begin{aligned}
\left|B_{K}(o, 1)\right| & =\frac{2 \pi^{\frac{m}{2}}}{m \Gamma\left(\frac{m}{2}\right)} \int_{|x|<1}\left(\frac{1-|x|^{4}}{16}\right)^{\frac{m}{2}} d x \\
& =\frac{\pi^{\frac{m+2 n}{2}}}{m 4^{m} \Gamma\left(\frac{m}{2}\right) \Gamma(n)} B\left(\frac{m}{2}+1, \frac{n}{2}\right) .
\end{aligned}
$$

Together with (3.14) and (3.15), (1.2) follows easily by direct calculation.

\section{Proof of Theorem 1.1 for $m \geq 3$ odd And $d_{K}$ Metric}

The proof follows the idea for the case of Heisenberg group (see [10]). More precisely, we consider the Poisson semigroup on $\mathbb{H}(2 n, m), e^{-h \sqrt{-\Delta}} f=f * P_{h}$ $(h>0)$.

As

$$
P_{h}(x, t) \geq 0, \quad \forall(x, t) \in \mathbb{R}^{2 n} \times \mathbb{R}^{m}, \quad\left\|P_{h}\right\|_{1}=1, \quad \forall h>0
$$


by the Hopf-Dunford-Schwartz maximal ergodic theorem, one has

$$
\left|\left\{g ; \sup _{s>0} \frac{1}{s} \int_{0}^{s} e^{-h \sqrt{-\Delta}} f(g) d h>\lambda\right\}\right| \leq \frac{2}{\lambda}\|f\|_{1}, \quad \forall \lambda>0, f \in L^{1}(\mathbb{H}(2 n, m)) .
$$

To prove Theorem 1.1 for $M=M_{K}$, one needs only to prove that there exists a constant $A>0$ such that for all $n, m \in \mathbb{N}^{*}$ verifying $m^{2} \ll \log n$, one has

$$
M_{K} f(g) \leq A n \sup _{s>0} \frac{1}{s} \int_{0}^{s} e^{-h \sqrt{-\Delta}} f(g) d h, \quad \forall g \in \mathbb{H}(2 n, m), 0 \leq f \in L^{1}(\mathbb{H}(2 n, m)) .
$$

For $g_{o} \in \mathbb{H}(2 n, m)$, denote $L_{g_{o}}$ the left translation operator defined by $L_{g_{o}} f(g)=$ $f\left(g_{o} g\right)$; one has $L_{g_{o}} M=M L_{g_{o}}\left(M=M_{k}\right.$ or $\left.M=M_{C C}\right)$ and $L_{g_{o}} e^{-h \sqrt{-\Delta}}=$ $e^{-h \sqrt{-\Delta}} L_{g_{o}}$. By the dilation structure on $\mathbb{H}(2 n, m)$, it suffices to find some $s(n)>0$ such that for all $g=(x, t) \neq o$, one has

$$
\begin{aligned}
\left|B_{K}(o, 1)\right|^{-1} \chi_{B_{K}(o, 1)}(g) & \leq A n \frac{1}{s(n)} \int_{0}^{s(n)} P_{h}(g) d h \\
& =A n \frac{1}{s(n)} \int_{0}^{s(n)} h^{-Q} P\left(\frac{x}{h}, \frac{t}{h^{2}}\right) d h \\
& =A n \frac{1}{s(n)} \int_{0}^{s(n)} h^{-Q} P\left(n, m, \frac{|x|^{2}}{h^{2}}, \frac{|t|}{h^{2}}\right) d h .
\end{aligned}
$$

To this end, we shall divide the proof into two cases: a) $\sqrt{n} \phi \geq C^{*} m$ with $\phi$ defined by (4.2) below and $C^{*} \gg 1$ to be determined later; b) $\sqrt{n} \phi<C^{*} m$. For case a) we adapt the method in [10 to prove (4.1). The key point here is to get the uniform lower bound for the Poisson kernel. For case b) we adapt the method in [12] to prove (4.1). The key point here is to get good estimations for $(-\Delta)^{-\frac{1}{2}}\left(g, g^{\prime}\right)$ and $(-\Delta)^{-\frac{1}{2}} \exp \left(-\omega \frac{d_{K}\left(g, g^{\prime}\right)}{\sqrt{n}} \sqrt{-\Delta}\right)\left(g, g^{\prime}\right)$.

For $M=M_{C C}$, we will prove the desired result by comparing with the case of $M_{K}$.

4.1. Lower bounds for the Poisson kernel $P(x, t)$. In what follows, one denotes $\phi \in\left[0, \frac{\pi}{2}\right]$ satisfying

$$
e^{-i \phi}=d_{K}^{-2}(x, t)\left(|x|^{2}-i 4|t|\right) .
$$

For $\omega_{1}, \omega_{2} \geq 0, r \in \mathbb{C}$, let

$$
f\left(\omega_{1}, \omega_{2}, r\right):=\frac{\omega_{1}^{2}}{4} r \operatorname{coth} r-i\left(\omega_{2} r\right) .
$$

For any $(x, t) \in \mathbb{H}(2 n, m)$, we have that $\Re f(|x|,|t|, r) \geq 0$ holds for all $0 \leq \Im r \leq$ $\frac{\pi}{2}$. In fact, for all $0 \leq \varpi \leq \frac{\pi}{2}$ and for $\iota \in \mathbb{R}$, one has (see [2], p. 645)

$$
\Re f(|x|,|t|, i \varpi)=\frac{\varpi}{\sin \varpi} \cos \varpi \frac{|x|^{2}}{4}+|t| \varpi \geq 0
$$

and

$$
\begin{aligned}
& \Re\{f(|x|,|t|, \iota+i \varpi)-f(|x|,|t|, i \varpi)\} \\
& =\frac{|x|^{2}}{4} \frac{\sinh ^{2} \iota}{\sinh ^{2} \iota+\sin ^{2} \varpi}(\iota \operatorname{coth} \iota-\varpi \cot \varpi) \geq 0 .
\end{aligned}
$$


Let

$$
F(r)=r^{\frac{m-1}{2}-k}\left(\frac{r}{\sinh r}\right)^{n}\left(\frac{1}{4}+\frac{|x|^{2}}{4} r \operatorname{coth} r-i|t| r\right)^{k-n-\frac{m}{2}-1} .
$$

It is clear that $F$ is analytic in

$$
\Omega=\left\{r \in \mathbb{C} ; 0<\Im r<\frac{\pi}{2}\right\},
$$

and continuous on $\bar{\Omega}$. Moreover, one has

$$
\lim _{r \in \bar{\Omega},|r| \longrightarrow+\infty}|F(r)|=0 .
$$

By Cauchy's fundamental theorem, one gets

$$
\int_{\mathbb{R}} F(r) d r=\int_{\mathbb{R}} F(r+i \phi) d r=W .
$$

By (4.2), it follows that

Denote

$$
f(|x|,|t|, r)=\frac{r}{4 \sinh r} d_{K}^{2}(x, t) \cosh (r-i \phi) .
$$

$$
\begin{aligned}
\mathrm{K} & =(r+i \phi)^{\frac{m-1}{2}-k}\left(\frac{\sinh (r+i \phi)}{r+i \phi}\right)^{\frac{m}{2}+1-k} \\
& \times\left(\frac{1}{4} \frac{\sinh (r+i \phi)}{r+i \phi}+\frac{1}{4} d_{K}^{2}(x, t) \cosh r\right)^{k-n-\frac{m}{2}-1} ;
\end{aligned}
$$

it follows that

$$
W=\int_{\mathbb{R}} \mathrm{K} d r
$$

Give

$$
\sigma=\sigma(m)>0
$$

which will be determined later (we remark here that we can choose $\sigma=m^{-\frac{1}{2}}$ ). Let

$$
W_{1}=\int_{-\sigma}^{\sigma} \mathrm{K} d r, \quad W_{2}=\int_{|r| \geq \sigma} \mathrm{K} d r
$$

We have $W=W_{1}+W_{2}$. First let us estimate $W_{2}$. Note that

$$
\max \left\{|\sinh (r+i \phi)|,\left|\frac{\sinh (r+i \phi)}{r+i \phi}\right|\right\} \leq \cosh r, \quad \forall 0 \leq \phi \leq \frac{\pi}{2}, r \in \mathbb{R} .
$$

Hence, as $n \longrightarrow+\infty$ and $d_{K}(x, t) \gg n^{\frac{1}{2}}$, one has

$$
\begin{aligned}
\left|W_{2}\right| & \leq 4^{n+\frac{m}{2}+1-k} \int_{|r| \geq \sigma}\left[\left(d_{K}^{2}(x, t)-1\right) \cosh r\right]^{k-n-\frac{m}{2}-1}(\cosh r)^{\frac{m}{2}+1-k} d r \\
& \leq 2 \cdot 4^{n+\frac{m}{2}+1-k}\left(d_{K}^{2}(x, t)-1\right)^{k-n-\frac{m}{2}-1} \int_{\sigma}^{+\infty}(\cosh r)^{-n} \sinh r(\sinh \sigma)^{-1} d r \\
& =\frac{2 \cdot 4^{n+\frac{m}{2}+1-k}}{d_{K}(x, t)^{2\left(n+\frac{m}{2}+1-k\right)}} e^{-\left(n+\frac{m}{2}+1-k\right) \ln \left(1-d_{K}^{-2}(x, t)\right)} \frac{(\cosh \sigma)^{1-n}}{n-1}(\sinh \sigma)^{-1} \\
& =\frac{2 \cdot 4^{n+\frac{m}{2}+1-k}}{d_{K}(x, t)^{2\left(n+\frac{m}{2}+1-k\right)}} \frac{(\cosh \sigma)^{1-n}}{n-1}(\sinh \sigma)^{-1}\left(1+O\left(\frac{n}{d_{K}^{2}(x, t)}\right)\right) .
\end{aligned}
$$

Now let us estimate $W_{1}$ : 
By Taylor's formula, we have

$$
\begin{aligned}
& \mid\left(\frac{\sinh (r+i \phi)}{r+i \phi}\right)^{\frac{m}{2}+1-k}-\left(\frac{\sin \phi}{\phi}\right)^{\frac{m}{2}+1-k} \\
& \quad-\left.\left(\frac{m}{2}+1-k\right) r\left(\frac{\sin \phi}{\phi}\right)^{\frac{m}{2}-k} \frac{d}{d s}\right|_{s=0}\left(\frac{\sinh (s+i \phi)}{s+i \phi}\right) \mid \\
& \leq \frac{r^{2}}{2} \sup _{\eta \in[0, r]}\left|\frac{d^{2}}{d \eta^{2}}\left(\frac{\sinh (\eta+i \phi)}{\eta+i \phi}\right)^{\frac{m}{2}+1-k}\right| .
\end{aligned}
$$

Note that there exists a constant $C>0$ such that for all $-m^{-\frac{1}{2}} \leq \eta \leq m^{-\frac{1}{2}}$, one has

$$
(\cosh \eta)^{\frac{m}{2}}=e^{\frac{m}{2} \ln [1+(\cosh \eta-1)]} \leq e^{\frac{m}{2}(\cosh \eta-1)} \leq C .
$$

Thus, by (4.4), there exists a constant $C_{1}>0$ such that for all $m \geq 3$ odd, $0 \leq k \leq \frac{m-3}{2}, 0 \leq \phi \leq \frac{\pi}{2}$ and $-m^{-\frac{1}{2}} \leq r \leq m^{-\frac{1}{2}}$, one has

$$
\begin{aligned}
\left(\frac{\sinh (r+i \phi)}{r+i \phi}\right)^{\frac{m}{2}+1-k} & =\left(\frac{\sin \phi}{\phi}\right)^{\frac{m}{2}+1-k}-i\left[\left(\frac{m}{2}+1-k\right) r\left(\frac{\sin \phi}{\phi}\right)^{\frac{m}{2}-k}\left(\frac{\sin \phi}{\phi}\right)^{\prime}\right] \\
& +E(m, k, \phi ; r)
\end{aligned}
$$

and

$$
|E(m, k, \phi ; r)| \leq C_{1} m^{2} r^{2} .
$$

Moreover, in the case of $\frac{n}{d_{K}^{2}(x, t)} \ll 1$, by the fact that $m<3 \log _{2}(2 n)$, one has

$$
\begin{aligned}
& \left(\frac{1}{4} \frac{\sinh (r+i \phi)}{r+i \phi}+\frac{1}{4} d_{K}^{2}(x, t) \cosh r\right)^{k-n-\frac{m}{2}-1} \\
& =4^{n+\frac{m}{2}+1-k}\left(d_{K}^{2}(x, t) \cosh r\right)^{k-n-\frac{m}{2}-1} e^{-\left(n+\frac{m}{2}+1-k\right) \ln \left(1+\frac{1}{d_{K}^{2}(z, t) \cosh r} \frac{\sinh (r+i \phi)}{r+i \phi}\right)} \\
& =4^{n+\frac{m}{2}+1-k} d_{K}(x, t)^{-2\left(n+\frac{m}{2}+1-k\right)}(\cosh r)^{k-n-\frac{m}{2}-1} S,
\end{aligned}
$$

where

$$
S=e^{-\left(n+\frac{m}{2}+1-k\right) \ln \left(1+\frac{1}{d_{K}^{2}(z, t) \cosh r} \frac{\sinh (r+i \phi)}{r+i \phi}\right)}=1+O\left(\frac{n}{d_{K}^{2}(x, t)}\right) .
$$

For $0 \leq j \leq \frac{m-1}{2}-k$, set

$$
\begin{aligned}
W_{1,1}(j) & =\left(\frac{\sin \phi}{\phi}\right)^{\frac{m}{2}+1-k} \int_{-\sigma}^{\sigma} r^{\frac{m-1}{2}-k-j}(\cosh r)^{k-n-\frac{m}{2}-1} S d r \\
W_{1,2}(j) & =-i\left[\left(\frac{m}{2}+1-k\right)\left(\frac{\sin \phi}{\phi}\right)^{\frac{m}{2}-k}\left(\frac{\sin \phi}{\phi}\right)^{\prime}\right] \\
& \times \int_{-\sigma}^{\sigma} r^{\frac{m-1}{2}-k-j+1}(\cosh r)^{k-n-\frac{m}{2}-1} S d r \\
W_{1,3}(j) & =\int_{-\sigma}^{\sigma} r^{\frac{m-1}{2}-k-j} E(m, k, \phi ; r)(\cosh r)^{k-n-\frac{m}{2}-1} S d r .
\end{aligned}
$$


One can write

$$
\begin{aligned}
W_{1} & =4^{n+\frac{m}{2}+1-k} d_{K}(x, t)^{-2\left(n+\frac{m}{2}+1-k\right)} \\
& \times \sum_{j=0}^{\frac{m-1}{2}-k} C_{\frac{m-1}{2}-k}^{j}(i \phi)^{j}\left(W_{1,1}(j)+W_{1,2}(j)+W_{1,3}(j)\right) .
\end{aligned}
$$

Let us first estimate $W_{1,1}\left(\frac{m-1}{2}-k\right)$ :

Note that

$$
\begin{aligned}
W_{1,1}\left(\frac{m-1}{2}-k\right) & =2\left(\frac{\sin \phi}{\phi}\right)^{\frac{m}{2}+1-k}\left[1+O\left(\frac{n}{d_{K}^{2}(x, t)}\right)\right] \\
& \times\left\{\int_{0}^{+\infty}-\int_{\sigma}^{+\infty}\right\}(\cosh r)^{k-n-\frac{m}{2}-1} d r .
\end{aligned}
$$

Combining this with the facts that

$$
\begin{aligned}
\int_{0}^{+\infty}(\cosh r)^{k-n-\frac{m}{2}-1} d r & =\int_{0}^{+\infty}(\cosh r)^{k-n-\frac{m}{2}-2} d(\sinh r) \\
& =\frac{1}{2} B\left(\frac{n+\frac{m}{2}+2-k}{2}-\frac{1}{2}, \frac{1}{2}\right), \quad \text { (see [16], pp. 6-7), } \\
\int_{\sigma}^{+\infty}(\cosh r)^{k-n-\frac{m}{2}-1} d r & \leq \frac{1}{\sinh \sigma} \int_{\sigma}^{+\infty}(\cosh r)^{k-n-\frac{m}{2}-1} \sinh r d r \\
& =\frac{1}{n+\frac{m}{2}-k} \frac{1}{\sinh \sigma}(\cosh \sigma)^{k-n-\frac{m}{2}}
\end{aligned}
$$

and

$$
\begin{gathered}
B\left(\frac{n+\frac{m}{2}+1-k}{2}, \frac{1}{2}\right)=\Gamma\left(\frac{1}{2}\right) \frac{\Gamma\left(\frac{n+\frac{m}{2}+1-k}{2}\right)}{\Gamma\left(\frac{n+\frac{m}{2}+2-k}{2}\right)}=\sqrt{\pi}\left(\frac{n+\frac{m}{2}+2-k}{2}\right)^{-\frac{1}{2}}(1+o(1)), \\
n \longrightarrow+\infty \quad \text { (see [16], p. 6 and p. 12) }
\end{gathered}
$$

one has

$$
\begin{aligned}
W_{1,1}\left(\frac{m-1}{2}-k\right) & =B\left(\frac{n+\frac{m}{2}+1-k}{2}, \frac{1}{2}\right)\left(\frac{\sin \phi}{\phi}\right)^{\frac{m}{2}+1-k} \\
& \times\left[1+O\left(\frac{1}{\sqrt{n} \sigma}\right)\right]\left[1+O\left(\frac{n}{d_{K}^{2}(x, t)}\right)\right], \quad \frac{d_{K}^{2}(x, t)}{n} \gg 1, n \gg 1 .
\end{aligned}
$$

On the other hand, observe that for $1 \leq \alpha \leq \frac{m-1}{2}+2$,

$$
\begin{aligned}
& \int_{-\sigma}^{\sigma}\left|r^{\alpha}\right|(\cosh r)^{k-n-\frac{m}{2}-1}|S| d r \\
\leq & 2\left[1+O\left(\frac{n}{d_{K}^{2}(x, t)}\right)\right] \int_{0}^{+\infty} \sinh ^{\alpha} r(\cosh r)^{k-n-\frac{m}{2}-1} d r \\
= & B\left(\frac{\alpha+1}{2}, \frac{n+\frac{m}{2}+1-k-\alpha}{2}\right)\left[1+O\left(\frac{n}{d_{K}^{2}(x, t)}\right)\right] .
\end{aligned}
$$


Together with (2.7), (4.7) and the fact $0 \leq \phi \leq \frac{\pi}{2}$, one has for $0 \leq j \leq \frac{m-1}{2}-k$, with the notation $\alpha(j):=\frac{m-1}{2}-k-j$,

$$
\begin{aligned}
& \left|W_{1,2}(j)\right|=W_{1,1}\left(\frac{m-1}{2}-k\right) m 2^{\frac{\alpha(j)+2}{2}} \Gamma\left(\frac{\alpha(j)+2}{2}\right) n^{-\frac{\alpha(j)+1}{2}} O(1), \\
& \left|W_{1,3}(j)\right|=W_{1,1}\left(\frac{m-1}{2}-k\right) m^{2}\left(\frac{\phi}{\sin \phi}\right)^{\frac{m}{2}} 2^{\frac{\alpha(j)+3}{2}} \Gamma\left(\frac{\alpha(j)+3}{2}\right) n^{-\frac{\alpha(j)+2}{2}} O(1),
\end{aligned}
$$

and for $0 \leq j \leq \frac{m-1}{2}-k-1$,

$$
\left|W_{1,1}(j)\right|=W_{1,1}\left(\frac{m-1}{2}-k\right) 2^{\frac{\alpha(j)+1}{2}} \Gamma\left(\frac{\alpha(j)+1}{2}\right) n^{-\frac{\alpha(j)}{2}} O(1),
$$

and $O(1) \leq C_{2}$ independent of $(2 n, m, k, j)$.

Note that

$$
m \leq 2 \log _{2}(2 n)+4,
$$

as $n \rightarrow+\infty$. It follows that

$$
\frac{m}{\sqrt{n}}=o(1)
$$

and for $0 \leq \phi \leq \frac{\pi}{2}$, one has

$$
\frac{m^{2}\left(\frac{\phi}{\sin \phi}\right)^{\frac{m}{2}}}{2 n} \leq \frac{m^{2}\left(\frac{\pi}{2}\right)^{\frac{m}{2}}}{2 n}=2^{2 \log _{2} m+\frac{m}{2} \log _{2}\left(\frac{\pi}{2}\right)-\log _{2}(2 n)}=o(1) .
$$

Thus, one has for $0 \leq j \leq \frac{m-1}{2}-k-1$,

$$
\begin{aligned}
& \left|W_{1,1}(j)\right|+\left|W_{1,2}(j)\right|+\left|W_{1,3}(j)\right| \\
& =W_{1,1}\left(\frac{m-1}{2}-k\right) 2^{\frac{\alpha(j)+1}{2}} \frac{\alpha(j)+1}{2} \Gamma\left(\frac{\alpha(j)+1}{2}\right) n^{-\frac{\alpha(j)}{2}} O(1)
\end{aligned}
$$

and

$$
\begin{aligned}
& W_{1,1}\left(\frac{m-1}{2}-k\right)+W_{1,2}\left(\frac{m-1}{2}-k\right)+W_{1,3}\left(\frac{m-1}{2}-k\right) \\
& =W_{1,1}\left(\frac{m-1}{2}-k\right)(1+o(1)) .
\end{aligned}
$$

Recall that (see for example [16], p. 3)

$$
\Gamma(2 r)=\pi^{-\frac{1}{2}} 2^{2 r-1} \Gamma(r) \Gamma\left(\frac{1}{2}+r\right) .
$$

One has for $0 \leq j \leq \frac{m-1}{2}-k-1$,

$$
\begin{aligned}
& C_{\frac{m-1}{2}-k}^{j} \phi^{j} 2^{\frac{\alpha(j)+1}{2}} \frac{\alpha(j)+1}{2} \Gamma\left(\frac{\alpha(j)+1}{2}\right) n^{-\frac{\alpha(j)}{2}} \\
= & \frac{\left(\frac{m-1}{2}-k\right) !}{j !} \frac{2^{\frac{\alpha(j)+1}{2}} \frac{\alpha(j)+1}{2} \Gamma\left(\frac{\alpha(j)+1}{2}\right)}{\Gamma\left(2 \frac{\alpha(j)+1}{2}\right)} n^{-\frac{m-1}{2}-k}(\sqrt{n} \phi)^{j} \\
= & \frac{\left(\frac{m-1}{2}-k\right) !}{j !} n^{-\frac{m-1}{2}-k}(\sqrt{n} \phi)^{j} \sqrt{\pi} \frac{1+\alpha(j)}{\Gamma\left(\frac{\alpha(j)}{2}+1\right)} 2^{-\frac{1+\alpha(j)}{2}} .
\end{aligned}
$$

Therefore, when

$$
\frac{\sqrt{n} \phi}{m} \gg 1
$$


holds and $\sigma=\frac{1}{\sqrt{m}}$, combined with (4.6) and (4.7), one has

$$
\begin{aligned}
W_{1} & =4^{n+\frac{m}{2}+1-k} d_{K}(x, t)^{-2\left(n+\frac{m}{2}+1-k\right)} W_{1,1}\left(\frac{m-1}{2}-k\right) \\
& \times(i \phi)^{\frac{m-1}{2}-k}\left[1+O\left(\frac{m}{\sqrt{n} \phi}\right)\right] \\
& =\sqrt{2 \pi} 4^{n+\frac{m}{2}+1-k} d_{K}(x, t)^{-2\left(n+\frac{m}{2}+1-k\right)}\left(\frac{\sin \phi}{\phi}\right)^{\frac{3}{2}} n^{-\frac{1}{2}}(i \sin \phi)^{\frac{m-1}{2}-k} \\
& \times(1+o(1))\left[1+O\left(\frac{m}{\sqrt{n} \phi}\right)\right]\left[1+O\left(\frac{n}{d_{K}^{2}(x, t)}\right)\right]
\end{aligned}
$$

Together with (3.11), (4.3) and the fact that $4|t|=d_{K}^{2}(x, t) \sin \phi$, we have the following

Proposition 4.1. We have

$$
\begin{aligned}
P(x, t) & =2^{\frac{3 m}{2}} \pi^{-n-\frac{m+1}{2}} d_{K}^{-Q-1}(x, t)\left(\frac{\sin \phi}{\phi}\right)^{\frac{3}{2}} n^{-\frac{1}{2}}(1+o(1))\left[1+O\left(\frac{m}{\sqrt{n} \phi}\right)\right] \\
& \times\left[1+O\left(\frac{n}{d_{K}^{2}(x, t)}\right)\right] \sum_{k=0}^{\frac{m-1}{2}} \frac{\Gamma\left(\frac{m-1}{2}+k\right)}{k ! \Gamma\left(\frac{m-1}{2}-k\right) 2^{k}}(\sin \phi)^{-2 k} \Gamma\left(n+\frac{m}{2}+1-k\right),
\end{aligned}
$$

for all $(x, t) \in \mathbb{H}(2 n, m)$ satisfying $\sqrt{n} \phi \gg m$ and $d_{K}^{2}(x, t) \gg n$, where $o(1) \rightarrow 0$ as $n \rightarrow \infty$. In particular, there exist two positive constants $C_{*}, C^{*} \gg 1$ such that for all $(x, t) \in \mathbb{H}(2 n, m)$ satisfying $\sqrt{n} \phi \geq C^{*} m$ and $d_{K}(x, t)>C_{*} n^{\frac{1}{2}}$, we have

$$
P(x, t) \geq 2^{-1} 2^{\frac{3 m}{2}} \pi^{-n-\frac{m+1}{2}} d_{K}^{-Q-1}(x, t)\left(\frac{\sin \phi}{\phi}\right)^{\frac{3}{2}} n^{-\frac{1}{2}} \Gamma\left(n+\frac{m}{2}+1\right) .
$$

4.2. Estimate of $(-\Delta)^{-\frac{1}{2}} \exp \left(-\omega \frac{d_{K}(g)}{\sqrt{n}} \sqrt{-\Delta}\right)(g)(\omega \geq 0)$ for $\sqrt{n} \phi \leq C^{*} m$. Throughout this subsection, we assume $\phi$ defined in (4.2) satisfies $\sqrt{n} \phi \leq C^{*} m$, which will be used repeatedly.

For $\omega \geq 0, g=(x, t) \in \mathbb{H}(2 n, m)$, one has

$$
\begin{aligned}
(-\Delta)^{-\frac{1}{2}} & \exp \left(-\omega d_{K}(g) n^{-\frac{1}{2}}(-\Delta)^{\frac{1}{2}}\right)(g) \\
& =\frac{1}{\sqrt{\pi}} \int_{0}^{\infty} h^{-1 / 2} e^{h \Delta}(g) \exp \left(-\frac{\omega^{2} d_{K}^{2}(g)}{4 n h}\right) d h .
\end{aligned}
$$

In particular,

$$
(-\Delta)^{-\frac{1}{2}}(g)=\frac{1}{\sqrt{\pi}} \int_{0}^{\infty} h^{-1 / 2} e^{h \Delta}(g) d h .
$$

Substituting (3.1) into (4.9) and applying the Fubini theorem, it follows that

$$
\begin{aligned}
(-\Delta)^{-\frac{1}{2}} & \exp \left(-\omega n^{-\frac{1}{2}} d_{K}(g) \sqrt{-\Delta}\right)(g) \\
& =2 \pi^{-\frac{1}{2}}(4 \pi)^{-n-\frac{m}{2}} \int_{0}^{\infty} r^{m-1}\left(\frac{r}{\sinh r}\right)^{n} \Xi d r
\end{aligned}
$$


where

$$
\begin{aligned}
\Xi & =\int_{0}^{\infty} h^{-n-m-\frac{1}{2}} \exp \left(-\frac{\left.|x|^{2} r \operatorname{coth} r+\omega^{2} \frac{d_{K}^{2}(g)}{n}\right)}{4 h}\right)\left(\frac{r|t|}{2 h}\right)^{-\frac{m-2}{2}} J_{\frac{m-2}{2}}\left(\frac{r|t|}{h}\right) d h \\
& =\left(\frac{r|t|}{2}\right)^{-\frac{m-2}{2}} \int_{0}^{\infty} \exp \left(-\frac{|x|^{2} r \operatorname{coth} r+\omega^{2} \frac{d_{K}^{2}(g)}{n}}{4} u\right) u^{n+\frac{m}{2}-\frac{1}{2}} J_{\frac{m-2}{2}}(r|t| u) d u .
\end{aligned}
$$

Thanks to (2.5), one has

$$
\Xi=\left(\frac{r|t|}{2}\right)^{-\frac{m-2}{2}} \Gamma\left(n+m-\frac{1}{2}\right) V^{-\left(n+\frac{m}{2}+\frac{1}{2}\right)} P_{n+\frac{m}{2}-\frac{1}{2}}^{-\frac{m-2}{2}}\left(\frac{U}{V}\right),
$$

where

$$
U=\frac{|x|^{2} r \operatorname{coth} r+\omega^{2} \frac{d_{K}^{2}(g)}{n}}{4}, V=\sqrt{U^{2}+(r|t|)^{2}} .
$$

Substituting into (4.10), we have, for $g=(x, t) \neq o$,

$$
\begin{aligned}
& (-\Delta)^{-\frac{1}{2}} \exp \left(-\omega \frac{d_{K}(g)}{\sqrt{n}} \sqrt{-\Delta}\right)(g) \\
& =2 \pi^{-\frac{1}{2}}(4 \pi)^{-n-\frac{m}{2}} \Gamma\left(n+m-\frac{1}{2}\right) \\
& \times \int_{0}^{\infty} r^{m-1}\left(\frac{r}{\sinh r}\right)^{n}\left(\frac{r|t|}{2}\right)^{-\frac{m-2}{2}} V^{-\left(n+\frac{m}{2}+\frac{1}{2}\right)} P_{n+\frac{m}{2}-\frac{m}{2}}^{-\frac{m-2}{2}}\left(\frac{U}{V}\right) d r .
\end{aligned}
$$

Recall that (see (4.2)

$$
\cos \phi=d_{K}^{-2}(x, t)|x|^{2}, \quad \sin \phi=4 d_{K}^{-2}(x, t)|t| .
$$

This yields

$$
U=\frac{d_{K}^{2}(x, t) r \operatorname{coth} r \cos \phi}{4}+\frac{\omega^{2} d_{K}^{2}(x, t)}{4 n}
$$

and

$$
V=\sqrt{U^{2}+(r|t|)^{2}}=\frac{r d_{K}^{2}(x, t) \sqrt{\cos ^{2} \phi+\sinh ^{2} r+U_{\star}}}{4 \sinh r},
$$

where we denote

(4.14) $U_{\star}=\frac{\omega^{4} \sinh ^{2} r}{n^{2} r^{2}}+\frac{2 \omega^{2} \sinh r \cosh r \cos \phi}{n r}$ (which is increasing for $r \geq 0$ ).

Also, we denote

$$
U_{0}=\frac{\omega^{4}}{n^{2}}+\frac{2 \omega^{2} \cos \phi}{n}
$$

For $\vartheta \in\left[0, \frac{\pi}{2}\right]$ satisfying

$$
\cos \vartheta=\frac{U}{V}=\frac{\cosh r \cos \phi+\frac{\omega^{2} \sinh r}{n r}}{\sqrt{\cos ^{2} \phi+\sinh ^{2} r+U_{\star}}},
$$

this implies

$$
\sin \vartheta=\frac{\sinh r \sin \phi}{\sqrt{\cos ^{2} \phi+\sinh ^{2} r+U_{\star}}} .
$$


For simplicity, denote

$$
\begin{gathered}
R:=r^{m-1}\left(\frac{r}{\sinh r}\right)^{n}\left(\frac{r|t|}{2}\right)^{-\frac{m-2}{2}} V^{-\left(n+\frac{m}{2}+\frac{1}{2}\right)}, \\
M_{1}:=\int_{0}^{\zeta} R P_{n+\frac{m}{2}-\frac{1}{2}}^{-\frac{m-2}{2}}(\cos \vartheta) d r,
\end{gathered}
$$

and

$$
M_{2}:=\int_{\zeta}^{\infty} R P_{n+\frac{m}{2}-\frac{1}{2}}^{-\frac{m-2}{2}}(\cos \vartheta) d r
$$

where $\zeta=n^{\nu_{0}-\frac{1}{2}}$ with $\nu_{0} \in\left(0, \frac{1}{2}\right)$; we shall find $\nu_{0}=\frac{1}{4}$ works. To estimate $M_{1}, M_{2}$, we need the following estimations:

4.2.1. Estimate of $P_{n+\frac{m}{2}-\frac{1}{2}}^{-\frac{m-2}{2}}(\cos \theta)\left(m \geq 3,0 \leq \theta \leq \frac{\pi}{2}\right)$ for $m \sin ^{2} \theta \leq 1$. The main result in this subsection is the following lemma.

Lemma 4.2. There exists some function $E(n, m ; \theta)=O\left(\frac{m}{\Gamma\left(\frac{m-1}{2}\right)}(\sin \theta)^{\frac{m}{2}+1}\right)$, such that for all $m \geq 3$ and $0 \leq \theta \leq 1$ satisfying $m \sin ^{2} \theta \leq 1$, we have

$$
P_{n+\frac{m}{2}-\frac{1}{2}}^{-\frac{m-2}{2}}(\cos \theta)=\left(n+\frac{m}{2}\right)^{-\frac{m-2}{2}} J_{\frac{m-2}{2}}\left(\left(n+\frac{m}{2}\right) \sin \theta\right)+E(n, m ; \theta) .
$$

Proof. Recall that for $m \geq 2$, we have (see [16]. p. 188 or [6], p. 961):

$$
\begin{aligned}
& \Gamma\left(\frac{m-1}{2}\right) P_{n+\frac{m}{2}-\frac{1}{2}}^{-\frac{m-2}{2}}(\cos \theta) \\
& =\left(\frac{\pi}{2}\right)^{-1 / 2}(\sin \theta)^{-\frac{m-2}{2}} \int_{0}^{\theta}(\cos t-\cos \theta)^{\frac{m-3}{2}} \cos \left[\left(n+\frac{m}{2}\right) t\right] d t \\
& =\left(\frac{\pi}{2}\right)^{-1 / 2}(\sin \theta)^{-\frac{m-2}{2}} 2^{\frac{m-3}{2}} \int_{0}^{\theta}\left(\sin ^{2} \frac{\theta}{2}-\sin ^{2} \frac{t}{2}\right)^{\frac{m-3}{2}} \cos \left[\left(n+\frac{m}{2}\right) t\right] d t .
\end{aligned}
$$

By change of the variable with $t=y \sin \theta$, one has

$$
\begin{aligned}
\Gamma\left(\frac{m-1}{2}\right) P_{n+\frac{m}{2}-\frac{1}{2}}^{-\frac{m-2}{2}}(\cos \theta) & =\left(\frac{\pi}{2}\right)^{-1 / 2}(\sin \theta)^{-\frac{m-4}{2}} 2^{\frac{m-3}{2}}\left(\sin \frac{\theta}{2}\right)^{m-3} \\
& \times \int_{0}^{\frac{\theta}{\sin \theta}}\left(1-\frac{\sin ^{2} \frac{y \sin \theta}{2}}{\sin ^{2} \frac{\theta}{2}}\right)^{\frac{m-3}{2}} \cos \left(\left(n+\frac{m}{2}\right) y \sin \theta\right) d y \\
& :=\left(\frac{\pi}{2}\right)^{-1 / 2}(\sin \theta)^{-\frac{m-4}{2}} 2^{\frac{m-3}{2}}\left(\sin \frac{\theta}{2}\right)^{m-3} L .
\end{aligned}
$$

To estimate $P_{n+\frac{m}{2}-\frac{1}{2}}^{-\frac{m-2}{2}}(\cos \theta)$, one needs only to estimate $L$. To this end, let

$$
L_{1}=\int_{0}^{1}\left(1-\frac{\sin ^{2} \frac{y \sin \theta}{2}}{\sin ^{2} \frac{\theta}{2}}\right)^{\frac{m-3}{2}} \cos \left(\left(n+\frac{m}{2}\right) y \sin \theta\right) d y
$$

and

$$
L_{2}=\int_{1}^{\frac{\theta}{\sin \theta}}\left(1-\frac{\sin ^{2} \frac{y \sin \theta}{2}}{\sin ^{2} \frac{\theta}{2}}\right)^{\frac{m-3}{2}} \cos \left(\left(n+\frac{m}{2}\right) y \sin \theta\right) d y
$$


thus

$$
L=L_{1}+L_{2}
$$

Note that one has

$$
\left|L_{2}\right| \leq \frac{\theta}{\sin \theta}-1=O\left(\sin ^{2} \theta\right), \quad \forall 0<\theta \leq \frac{\pi}{2}, \quad \forall(2 n, m) .
$$

For $0 \leq y \leq 1$, by the differential mean value theorem, we have

$$
\left(1-\frac{\sin ^{2} \frac{y \sin \theta}{2}}{\sin ^{2} \frac{\theta}{2}}\right)^{(m-3) / 2}=\left(1-y^{2}\right)^{(m-3) / 2}+E_{1}(m, y, \theta),
$$

where $E_{1}(m, y, \theta)=O\left(m \sin ^{2} \theta\right)$. Hence

$$
\begin{aligned}
L_{1} & =\int_{0}^{1}\left(1-y^{2}\right)^{(m-3) / 2} \cos \left(\left(n+\frac{m}{2}\right) y \sin \theta\right) d y \\
& +\int_{0}^{1} E_{1}(m, y, \theta) \cos \left(\left(n+\frac{m}{2}\right) y \sin \theta\right) d y \\
& =\frac{1}{2} \pi^{1 / 2}\left(\frac{\left(n+\frac{m}{2}\right) \sin \theta}{2}\right)^{-\frac{m-2}{2}} \Gamma\left(\frac{m-1}{2}\right) J_{\frac{m-2}{2}}\left(\left(n+\frac{m}{2}\right) \sin \theta\right) \\
& +E_{2}\left(m, \theta, n+\frac{m}{2}\right),
\end{aligned}
$$

with $E_{2}\left(m, \theta, n+\frac{m}{2}\right)=O\left(m \sin ^{2} \theta\right)$, where the last equality follows from (2.1).

For $m \geq 3$, combining this with (4.22), (4.23), (4.24) and (4.25), one has

$$
\begin{aligned}
P_{n+\frac{m}{2}-\frac{1}{2}}^{-\frac{m-2}{2}}(\cos \theta) & =\left(n+\frac{m}{2}\right)^{-\frac{m-2}{2}} J_{\frac{m-2}{2}}\left(\left(n+\frac{m}{2}\right) \sin \theta\right) \\
& +O\left(\frac{2^{-\frac{m-3}{2}} m}{\Gamma\left(\frac{m-1}{2}\right)}(\sin \theta)^{\frac{m}{2}+1}\left(\cos \frac{\theta}{2}\right)^{3-m}\right) \\
& +\left(n+\frac{m}{2}\right)^{-\frac{m-2}{2}}\left[\left(\cos \frac{\theta}{2}\right)^{3-m}-1\right] J_{\frac{m-2}{2}}\left(\left(n+\frac{m}{2}\right) \sin \theta\right) .
\end{aligned}
$$

Notice that for $0<m \theta^{2} \leq 1$,

$$
1 \leq\left(\cos \frac{\theta}{2}\right)^{3-m} \leq e^{-m \ln \left(1-2 \sin ^{2} \frac{\theta}{4}\right)}
$$

By (2.2), we get the desired result.

4.2.2. Estimates of $M_{1}$ and $M_{2}$. Our aim in this subsection is now to estimate $M_{1}$ and $M_{2}$.

Notice that for $0 \leq \phi \leq C^{*} \frac{m}{\sqrt{n}}$ and $0<r \leq \zeta=n^{\nu_{0}-\frac{1}{2}}$, by (4.17), we have for $n$ large enough,

$$
m \sin ^{2} \vartheta \leq 1
$$

Substituting (4.21) into the expression (4.19), we have

$$
\begin{aligned}
M_{1} & =\int_{0}^{\zeta} R\left[\left(n+\frac{m}{2}\right)^{-\frac{m-2}{2}} J_{\frac{m-2}{2}}\left(\left(n+\frac{m}{2}\right) \sin \vartheta\right)+E(n, m ; \vartheta)\right] d r \\
(4.26) & =\left(\frac{\sin \phi}{2}\right)^{-\frac{m-2}{2}} 4^{n+m-\frac{1}{2}} d_{K}^{-(2 n+2 m)+1}(x, t)\left(M_{11}+\left(n+\frac{m}{2}\right)^{-\frac{m-2}{2}} \cdot M_{12}\right),
\end{aligned}
$$


where

$$
M_{11}=\int_{0}^{\zeta} r^{\frac{m}{2}}\left(\frac{r}{\sinh r}\right)^{-\left(\frac{m}{2}+\frac{1}{2}\right)}\left(\cos ^{2} \phi+\sinh ^{2} r+U_{\star}\right)^{-\frac{1}{2}\left(n+\frac{m}{2}+\frac{1}{2}\right)} E(n, m ; \vartheta) d r
$$

and

$$
M_{12}=\int_{0}^{\zeta} r^{\frac{m}{2}}\left(\frac{r}{\sinh r}\right)^{-\left(\frac{m}{2}+\frac{1}{2}\right)} \frac{J_{\frac{m-2}{2}}\left(\left(n+\frac{m}{2}\right) \sin \vartheta\right)}{\left(\cos ^{2} \phi+\sinh ^{2} r+U_{\star}\right)^{\frac{1}{2}\left(n+\frac{m}{2}+\frac{1}{2}\right)}} d r .
$$

We have the following estimates for $M_{11}$ and $M_{12}$. The proof is postponed until the Appendix.

Lemma 4.3. Given $\omega \geq 0$ and $C^{*}>0$, there exists a constant $C\left(\omega, C^{*}\right)>0$ such that

$$
\begin{aligned}
M_{12} & =e^{-\omega^{2}}\left(\frac{\left(n+\frac{m}{2}\right) \sin \phi}{2}\right)^{\frac{1}{2}\left(n+m-\frac{5}{2}\right)} \frac{(\cos \phi)^{-\left(n-\frac{1}{2}\right)}}{\Gamma\left(\frac{1}{2}\left(n+m-\frac{1}{2}\right)\right)} \\
& \times K_{\frac{1}{2}\left(n-\frac{1}{2}\right)}\left(\left(n+\frac{m}{2}\right) \sin \phi\right)\left[1+O\left(n^{-1} e^{C\left(\omega, C^{*}\right) m^{2}}\right)\right]
\end{aligned}
$$

and

$$
\left|M_{11}\right| \leq\left(n+\frac{m}{2}\right)^{-\frac{m-2}{2}} M_{12} O\left(\frac{m^{\frac{3}{2}}}{n} e^{C\left(\omega, C^{*}\right) m^{2}}\right)
$$

Here $K_{\nu}(z)$ is the modified Bessel function defined by

$$
K_{\nu}(z)=K_{-\nu}(z)=\int_{0}^{\infty} e^{-z \cosh t} \cosh (\nu t) d t, \Re z>0 .
$$

Hence we obtain from (4.26),

$$
\begin{aligned}
M_{1} & =4^{n+m-\frac{1}{2}} e^{-\omega^{2}} d_{K}^{-Q+1}(x, t)\left(\frac{\left(n+\frac{m}{2}\right) \sin \phi}{2}\right)^{\frac{1}{2}\left(n-\frac{1}{2}\right)} \\
& \times \frac{(\cos \phi)^{-\left(n-\frac{1}{2}\right)}}{\Gamma\left(\frac{1}{2}\left(n+m-\frac{1}{2}\right)\right)} K_{\frac{1}{2}\left(n-\frac{1}{2}\right)}\left(\left(n+\frac{m}{2}\right) \sin \phi\right)\left[1+O\left(n^{-1} e^{C\left(\omega, C^{*}\right) m^{2}}\right)\right] .
\end{aligned}
$$

Furthermore one has the following estimate by (7.7) in the Appendix, for $m^{2} \ll$ $\log n$ :

$$
M_{1} \geq \frac{1}{9} e^{-\omega^{2}} 4^{n+m-\frac{1}{2}} d_{K}^{-Q+1}(x, t) \frac{\Gamma\left(\frac{1}{2}\left(n-\frac{1}{2}\right)\right)}{\Gamma\left(\frac{1}{2}\left(n+m-\frac{1}{2}\right)\right)} .
$$

Recall that

$$
M_{2}=\int_{\zeta}^{\infty} R P_{n+\frac{m}{2}-\frac{1}{2}}^{-\frac{m-2}{2}}(\cos \vartheta) d r
$$


(see (4.20) ), where $R$ is defined in (4.18). Combining with (2.6) and (4.14), we have

$$
\begin{aligned}
\left|M_{2}\right| & \leq 2^{\frac{m+2}{2}} 4^{n+m+\frac{1}{2}} d_{K}^{-Q+1}(x, t) \frac{\Gamma\left(n+\frac{3}{2}\right)}{\Gamma\left(n+\frac{m+1}{2}\right)} \pi^{-\frac{1}{2}}\left(n+\frac{m-1}{2}\right)^{-\frac{1}{2}}(\sin \phi)^{-m+\frac{3}{2}} \\
& \times \int_{\zeta}^{\infty} r^{-\frac{1}{2}} \sinh r\left(\cos ^{2} \phi+\sinh ^{2} r+U_{\star}\right)^{-\frac{1}{2}(n+1)} d r \\
& \leq c^{\prime} 2^{\frac{m+2}{2}} 4^{n+m+\frac{1}{2}} d_{K}^{-Q+1}(x, t) \frac{\Gamma\left(n+\frac{3}{2}\right)}{\Gamma\left(n+\frac{m+1}{2}\right)}\left(n+\frac{m}{2}\right)^{-\frac{1}{2}}(\sin \phi)^{-m+\frac{3}{2}} \\
& \times\left(\cos ^{2} \phi+U_{0}+\sinh ^{2} \zeta\right)^{-\frac{1}{2}(n-1)} \\
& \leq C\left(\omega, \nu_{0}\right) 2^{\frac{m+2}{2}} 4^{n+m+\frac{1}{2}} d_{K}^{-Q+1}(x, t) e^{-\omega^{2}}(\sin \phi)^{-m+\frac{3}{2}} e^{-\frac{1}{4} n^{2 \nu_{0}}},
\end{aligned}
$$

where the last inequality follows from (2.7) and

$$
\begin{aligned}
\left(\cos ^{2} \phi+U_{0}+\sinh ^{2} \zeta\right)^{-\frac{1}{2}\left(n-\frac{1}{2}\right)} & \leq e^{-\frac{n-1}{2} \ln \left[\left(\cos \phi+\frac{\omega^{2}}{n}\right)^{2}+\zeta^{2}\right]} \\
& \leq C\left(\omega, \nu_{0}\right) e^{-\omega^{2}} e^{-\frac{1}{4} n^{2 \nu_{0}}} .
\end{aligned}
$$

Notice that for $n$ large enough,

$$
\frac{\Gamma\left(\frac{1}{2}\left(n-\frac{1}{2}\right)\right)}{\Gamma\left(\frac{1}{2}\left(n+m-\frac{1}{2}\right)\right)} \sim\left(\frac{n}{2}\right)^{-\frac{m}{2}} .
$$

By (4.29) and (4.30), it follows, for some constant $C\left(\omega, C^{*}\right) \in \mathbb{R}^{+}$, that

$$
\left|M_{2}\right| \leq M_{1} O\left(e^{-C\left(\omega, C^{*}\right) n^{2 \nu_{0}}}\right) .
$$

Together with (4.29), (4.30) and choosing $\nu_{0}=\frac{1}{4}$, we have the following.

Proposition 4.4. Given $\omega \geq 0, C^{*}>0$. There exists a constant $c\left(\omega, C^{*}\right)>0$ such that for all $g=(x, t) \in \mathbb{H}(2 n, m)$ satisfying $\sqrt{n} \phi \leq C^{*} m$,

$$
\begin{aligned}
& (-\Delta)^{-\frac{1}{2}} \exp \left(-\omega \frac{d_{K}(g)}{\sqrt{n}} \sqrt{-\Delta}\right)(g) \\
& =2^{m} \pi^{-n-\frac{m}{2}-\frac{1}{2}} e^{-\omega^{2}} \Gamma\left(n+m-\frac{1}{2}\right) \\
& \times\left(d_{K}(g)\right)^{-(2 n+2 m)+1}\left(\frac{\left(n+\frac{m}{2}\right) \sin \phi}{2}\right)^{\frac{1}{2}\left(n-\frac{1}{2}\right)} \frac{(\cos \phi)^{-\left(n-\frac{1}{2}\right)}}{\Gamma\left(\frac{1}{2}\left(n+m-\frac{1}{2}\right)\right)} \\
& \times K_{\frac{1}{2}\left(n-\frac{1}{2}\right)}\left(\left(n+\frac{m}{2}\right) \sin \phi\right)\left[1+O\left(n^{-1} e^{c\left(\omega, C^{*}\right) m^{2}}\right)\right] .
\end{aligned}
$$

Moreover, if we assume $m^{2} \ll \log n$ holds, by (4.30), we have the following estimate:

$$
(-\Delta)^{-\frac{1}{2}}(g) \geq \frac{1}{9} 2^{m} \pi^{-\frac{2 n+m+1}{2}}\left(d_{K}(g)\right)^{1-(2 n+2 m)} \frac{\Gamma\left(\frac{1}{2}\left(n-\frac{1}{2}\right)\right) \Gamma\left(n+m-\frac{1}{2}\right)}{\Gamma\left(\frac{1}{2}\left(n+m-\frac{1}{2}\right)\right)}
$$

4.3. Proof of Theorem 1.1 for $m$ odd. First let us prove the following.

Lemma 4.5. There exists a constant $C>0$ such that for all $(2 n, m)$ satisfying $m^{2} \ll \log n$ with $m$ odd, we have

$$
\begin{gathered}
n^{\frac{3}{2}}\left|B_{K}(o, 1)\right|(-\Delta)^{-\frac{1}{2}}(g)>C, \quad \forall g=(x, t) \in B_{K}(o, 1) \backslash\{o\} \subset \mathbb{H}(2 n, m) \\
\text { satisfying } \sqrt{n} \phi \leq C^{*} m .
\end{gathered}
$$


Proof. Recall that (see (3.16)),

$$
\left|B_{K}(o, 1)\right|=\frac{\pi^{\frac{m+2 n}{2}}}{m 4^{m} \Gamma\left(\frac{m}{2}\right) \Gamma(n)} B\left(\frac{m}{2}+1, \frac{n}{2}\right) .
$$

Combining this with the estimate (4.34) and the property $\Gamma(r+1)=r \Gamma(r)(r>0)$, one has

$$
n^{\frac{3}{2}}\left|B_{K}(o, 1)\right|(-\Delta)^{-\frac{1}{2}}(g) \geq \frac{1}{18} \pi^{-\frac{1}{2}} 2^{-m} n^{\frac{3}{2}} \frac{\Gamma\left(m+n-\frac{1}{2}\right) \Gamma\left(\frac{1}{2}\left(n-\frac{1}{2}\right)\right) \Gamma\left(\frac{n}{2}\right)}{\Gamma\left(\frac{1}{2}\left(m+n-\frac{1}{2}\right)\right) \Gamma(n) \Gamma\left(\frac{n}{2}+\frac{m}{2}+1\right)} .
$$

Thanks to the property $\Gamma(2 z)=\pi^{-\frac{1}{2}} 2^{2 z-1} \Gamma(z) \Gamma\left(z+\frac{1}{2}\right)(z>0)$ (see [16], p. 3),

$$
\begin{aligned}
n^{\frac{3}{2}}\left|B_{K}(o, 1)\right|(-\Delta)^{-\frac{1}{2}}(g) & \geq \frac{\sqrt{2}}{36} \pi^{-\frac{1}{2}} n^{\frac{3}{2}} \frac{\Gamma\left(\frac{n}{2}-\frac{1}{4}\right) \Gamma\left(\frac{n}{2}+\frac{m}{2}+\frac{1}{4}\right)}{\Gamma\left(\frac{n}{2}+\frac{1}{2}\right) \Gamma\left(\frac{n}{2}+\frac{m}{2}+1\right)} \\
& \sim \frac{1}{9} \pi^{-\frac{1}{2}}\left(1+\frac{m+1}{n}\right)^{-\frac{3}{4}}
\end{aligned}
$$

the last inequality follows from (2.7). The desired conclusion follows.

Now we are in a position for the

Proof of (4.1). To prove the desired result, we divide it into two cases.

Case (a). $\sqrt{n} \phi>C^{*} m$. For $0<d_{K}(x, t)<1$, we have

$$
\begin{aligned}
& \int_{0}^{\frac{1}{C_{*} \sqrt{n}}} P_{h}(x, t) d h \\
& \geq \int_{0}^{\frac{d_{K}(x, t)}{C_{*} \sqrt{n}}} P_{h}(x, t) d h=\int_{0}^{\frac{d_{K}(x, t)}{C_{*} \sqrt{n}}} h^{-Q} P\left(\frac{x}{h}, \frac{t}{h^{2}}\right) d h \\
& \geq 2^{-1} 2^{\frac{3 m}{2}} \pi^{-n-\frac{m+1}{2}}\left(\frac{\sin \phi}{\phi}\right)^{\frac{3}{2}} n^{-\frac{1}{2}} \Gamma\left(n+\frac{m}{2}+1\right) \int_{0}^{\frac{d_{K}(x, t)}{C_{*} \sqrt{n}}} h^{-Q} d_{K}^{-Q-1}\left(\frac{x}{h}, \frac{t}{h^{2}}\right) d h,
\end{aligned}
$$

where the last inequality follows from Proposition 4.1. By the scaling property of Korányi norm $d_{K}$, it yields

$$
\begin{aligned}
& \int_{0}^{\frac{d_{K}(x, t)}{C_{*} \sqrt{n}}} P_{h}(x, t) d h \\
& \geq 2^{-2} 2^{\frac{3 m}{2}} \pi^{-n-\frac{m+1}{2}} n^{-\frac{3}{2}}\left(\frac{\sin \phi}{\phi}\right)^{\frac{3}{2}} \Gamma\left(n+\frac{m}{2}+1\right) d_{K}^{-Q+1}(x, t) C_{*}^{-2} .
\end{aligned}
$$

Together with (3.16), it follows for all $g=(x, t) \in B_{K}(o, 1)$ that

$$
\begin{aligned}
& n^{\frac{3}{2}}\left|B_{K}(o, 1)\right| \int_{0}^{\frac{1}{C_{*} \sqrt{n}}} P_{h}(x, t) d h \\
& \geq 2^{-3} \pi^{-\frac{1}{2}}\left(\frac{\sin \phi}{\phi}\right)^{\frac{3}{2}} C_{*}^{-2} 2^{-\frac{m}{2}} \frac{\Gamma\left(\frac{n}{2}\right)}{\Gamma\left(\frac{n}{2}+\frac{m}{2}+1\right)} \frac{\Gamma\left(n+\frac{m}{2}+1\right)}{\Gamma(n)} .
\end{aligned}
$$

Thanks to (2.7),

$$
n^{\frac{3}{2}}\left|B_{K}(o, 1)\right| \int_{0}^{\frac{1}{C_{*} \sqrt{n}}} P_{h}(x, t) d h \geq 2^{-4} \pi^{-\frac{1}{2}}\left(\frac{\sin \phi}{\phi}\right)^{\frac{3}{2}} C_{*}^{-2} .
$$


Choose $s(n)=\frac{1}{C_{*} \sqrt{n}} ;$ (4.1) follows directly.

Case (b). $\sqrt{n} \phi \leq C^{*} m$. Put $s(n)=\frac{100}{\sqrt{n}}$. By Proposition 4.4 there exists $c>0$ such that for $0<d_{K}(g)<1$, we have

$$
\begin{aligned}
\int_{0}^{\frac{100}{\sqrt{n}}} e^{-h \sqrt{-\Delta}}(g) d h & >\int_{0}^{\frac{100}{\sqrt{n}} d_{K}(g)} e^{-h \sqrt{-\Delta}}(g) d h \\
& =(-\Delta)^{-\frac{1}{2}}(g)-(-\Delta)^{-\frac{1}{2}} e^{-100 \frac{d_{K}(g)}{\sqrt{n}}(-\Delta)^{-\frac{1}{2}}}(g) \\
& \geq c(-\Delta)^{-\frac{1}{2}}(g) .
\end{aligned}
$$

Combining this with Lemma 4.5 it is clear that (4.1) holds.

In all, if we choose $s(n)=\frac{100}{\sqrt{n}}$, then (4.1) holds.

Remark 4.6. From the above proof, we remark that there exists a constant $c>0$ such that for $\log n \gg l^{2}$ with $l$ odd, we have $\forall 0<\left(|x|^{4}+16|t|^{2}\right)^{\frac{1}{4}}<4$,

$$
\begin{aligned}
& n^{\frac{3}{2}} \int_{0}^{\frac{400}{\sqrt{n}}} h^{-(2 n+2 l)} P\left(n, l, \frac{|x|^{2}}{h^{2}}, \frac{|t|}{h^{2}}\right) d h \\
& \geq c \pi^{-\frac{2 n+l}{2}} 4^{l} \frac{\Gamma(n) \Gamma\left(\frac{n}{2}+\frac{l}{2}+1\right)}{\Gamma\left(\frac{n}{2}\right)}\left[|x|^{4}+16|t|^{2}\right]^{\frac{1-(2 n+2 l)}{4}} .
\end{aligned}
$$

\section{Proof of Theorem 1.1 for $m$ Even AND $d_{K}$ Metric}

Proof of (4.1) for $m$ even. For $g=(x, t) \in \mathbb{H}(2 n, m)$ with $d_{K}(g)<1$ and $|t| \neq 0$, thanks to (3.8), one has

$$
\begin{aligned}
& n^{\frac{3}{2}} \int_{0}^{\frac{400}{\sqrt{n}}} h^{-(2 n+2 m)} P\left(n, m, \frac{|x|^{2}}{h^{2}}, \frac{|t|}{h^{2}}\right) d h \\
& =2|t| n^{\frac{3}{2}} \int_{0}^{\frac{400}{\sqrt{n}}} h^{-(2 n+2 m+2)} d h \int_{1}^{\infty} \frac{w}{\sqrt{w^{2}-1}} P\left(n, m+1, \frac{|x|^{2}}{h^{2}}, \frac{w|t|}{h^{2}}\right) d w \\
& >2|t| \int_{1}^{\frac{2}{|t|}} \frac{w}{\sqrt{w^{2}-1}}\left\{n^{\frac{3}{2}} \int_{0}^{\frac{400}{\sqrt{n}}} h^{-(2 n+2 m+2)} P\left(n, m+1, \frac{|x|^{2}}{h^{2}}, \frac{w|t|}{h^{2}}\right) d h\right\} d w \\
& \geq c \pi^{-\frac{2 n+m+1}{2}} 4^{m+1} \frac{\Gamma(n) \Gamma\left(\frac{n}{2}+\frac{m+1}{2}+1\right)}{\Gamma\left(\frac{n}{2}\right)}|t| \\
& \times \int_{1}^{\frac{2}{|t|}} \frac{w}{\sqrt{w^{2}-1}}\left[|x|^{4}+16(w|t|)^{2}\right]^{\frac{1-(2 n+2 m+2)}{4}} d w .
\end{aligned}
$$

However, by the change of variable $\sqrt{w^{2}-1}=s /|t|$, we have

$$
\begin{aligned}
& |t| \int_{1}^{\frac{2}{|t|}} \frac{w}{\sqrt{w^{2}-1}}\left[|x|^{4}+16(w|t|)^{2}\right]^{\frac{1-(2 n+2 m+2)}{4}} d w \\
& >\int_{0}^{1}\left[\left(|x|^{4}+16|t|^{2}\right)+s^{2}\right]^{\frac{1-(2 n+2 m+2)}{4}} d s \\
& =\left(|x|^{4}+16|t|^{2}\right)^{\frac{1-(2 n+2 m)}{4}} \int_{0}^{\left(|x|^{4}+16|t|^{2}\right)^{-\frac{1}{2}}}\left(1+\tau^{2}\right)^{\frac{1-(2 n+2 m+2)}{4}} d \tau .
\end{aligned}
$$


Observe that

$$
\begin{aligned}
& \int_{0}^{\left(|x|^{4}+16|t|^{2}\right)^{-\frac{1}{2}}}\left(1+\tau^{2}\right)^{\frac{1-(2 n+2 m+2)}{4}} d \tau \\
& >\int_{0}^{+\infty}\left(1+\tau^{2}\right)^{\frac{1-(2 n+2 m+2)}{4}} d \tau-\int_{1}^{+\infty} \tau\left(1+\tau^{2}\right)^{\frac{1-(2 n+2 m+2)}{4}} d \tau \\
& =B\left(\frac{1}{2}, \frac{n+m}{2}-\frac{1}{4}\right)-o\left((n+m)^{-2}\right) \\
& >\frac{1}{2} B\left(\frac{1}{2}, \frac{n+m}{2}-\frac{1}{4}\right), \text { for all } n+m \gg 1 .
\end{aligned}
$$

By Stirling's formula, we obtain (4.1) with $s(n)=\frac{400}{\sqrt{n}}$ for $m$ even immediately.

Remark 5.1. From the proof of Theorem 1.1, it is easy to see that (1.3) holds.

\section{Proof of Theorem 1.1 for $d_{C C}$ Metric}

We will need two lemmas as follows.

Lemma 6.1. For all $g=(x, t) \in \mathbb{H}(2 n, m)$, we have $d_{K}(g) \leq d_{C C}(g)$.

Proof. The proof follows from [10].

Lemma 6.2. There exists a constant $c>0$ (independent of $m$ and $n$ ) such that

$$
\left|B_{C C}(o, 1)\right| \geq c^{m}\left|B_{K}(o, 1)\right|, \text { for all } n \in \mathbb{N}^{*} \text {. }
$$

Proof (essential due to [10]). Clearly we have

$$
\left|B_{C C}(o, 1)\right|=\int_{\substack{\frac{\theta}{\sin \theta}|x|<1,-\pi<\theta<\pi, 4|t|=\mu(\theta)|x|^{2}}} d x d t=\Omega_{m} \int_{|x|<1}\left(\frac{\mu\left(\theta_{0}\right)|x|^{2}}{4}\right)^{m} d x,
$$

where $\theta_{0} \in[0, \pi)$ satisfies $\frac{\sin \theta_{0}}{\theta_{0}}=|x|$ and $\Omega_{m}=\frac{2 \pi^{\frac{m}{2}}}{m \Gamma\left(\frac{m}{2}\right)}$. It follows that

$$
\left|B_{C C}(o, 1)\right|=\Omega_{m} \int_{|x|<1}\left(\frac{2 \theta_{0}-\sin 2 \theta_{0}}{8 \theta_{0}^{2}}\right)^{m} d x
$$

Notice that

$$
\begin{aligned}
\left|B_{K}(o, 1)\right| & =\Omega_{m} \int_{|x|<1}\left(\frac{1-|x|^{4}}{16}\right)^{\frac{m}{2}} d x \\
& =\Omega_{m} \int_{|x|<1} \frac{1}{4^{m}}\left[1-\left(\frac{\sin \theta_{0}}{\theta_{0}}\right)^{4}\right]^{\frac{m}{2}} d x .
\end{aligned}
$$

Denote

$$
c:=\inf _{\theta_{0} \in[0, \pi)} \frac{2 \theta_{0}-\sin \left(2 \theta_{0}\right)}{2 \theta_{0}^{2}}\left(1-\left(\frac{\sin \theta_{0}}{\theta_{0}}\right)^{4}\right)^{-\frac{1}{2}},
$$

which is positive; see [10]. Combining with (6.1) and (6.2), we complete the proof.

Remark 6.3. By certain properties of the Bessel functions, we can improve the constant $c$. 
Proof of Theorem 1.1 for $M=M_{C C}$. For $m$ fixed, the desired conclusion follows directly from the one with $M=M_{K}$ and the above two lemmas.

\section{Appendix: Proof of Lemma 4.3}

Recall that $0 \leq \phi \ll 1, \omega \geq 0, \zeta=n^{\nu_{0}-\frac{1}{2}}$ with $\nu_{0} \in\left(0, \frac{1}{2}\right)$, which will be determined later, and

$$
\begin{aligned}
U_{\star} & =\frac{\omega^{4} \sinh ^{2} r}{n^{2} r^{2}}+\frac{2 \omega^{2} \sinh r \cosh r \cos \phi}{n r}, \quad \sin \vartheta=\frac{\sinh r \sin \phi}{\sqrt{\cos ^{2} \phi+\sinh ^{2} r+U_{\star}}}, \\
M_{12} & =\int_{0}^{\zeta} \frac{\sinh ^{\frac{m}{2}} r \sqrt{\frac{\sinh r}{r}} J_{\frac{m-2}{2}}\left(\left(n+\frac{m}{2}\right) \sin \vartheta\right)}{\left(\cos ^{2} \phi+\sinh ^{2} r+U_{\star}\right)^{\frac{1}{2}\left(n+\frac{m}{2}+\frac{1}{2}\right)}} d r .
\end{aligned}
$$

Let

$$
\begin{aligned}
\Theta & :=\left(\cos ^{2} \phi+\sinh ^{2} r+U_{\star}\right)^{-\frac{1}{2}\left(n+\frac{m}{2}+\frac{1}{2}\right)} J_{\frac{m-2}{2}}\left(\left(n+\frac{m}{2}\right) \sin \vartheta\right) \\
& \stackrel{\text { (4.17) }}{=}\left(\left(n+\frac{m}{2}\right) \sin \phi \sinh r\right)^{\frac{m-2}{2}}\left(\cos ^{2} \phi+\sinh ^{2} r+U_{\star}\right)^{-\frac{1}{2}\left(n+m-\frac{1}{2}\right)} \\
& \times\left(\frac{\left(n+\frac{m}{2}\right) \sin \phi \sinh r}{\sqrt{\cos ^{2} \phi+\sinh ^{2} r+U_{\star}}}\right)^{-\frac{m-2}{2}} J_{\frac{m-2}{2}}\left(\frac{\left(n+\frac{m}{2}\right) \sin \phi \sinh r}{\sqrt{\cos ^{2} \phi+\sinh ^{2} r+U_{\star}}}\right) \\
& =:\left(\left(n+\frac{m}{2}\right) \sin \phi \sinh r\right)^{\frac{m-2}{2}}\left(\cos ^{2} \phi+\sinh ^{2} r+U_{\star}\right)^{-\frac{1}{2}\left(n+m-\frac{1}{2}\right)} \cdot I_{2} .
\end{aligned}
$$

Applying the differential mean value theorem to the function $z \rightarrow z^{-\nu} J_{\nu}(z)$, one gets

$$
I_{2}=\left(\frac{\left(n+\frac{m}{2}\right) \sin \phi \sinh r}{\sqrt{\cos ^{2} \phi+U_{0}}}\right)^{-\frac{m-2}{2}} J_{\frac{m-2}{2}}\left(\frac{\left(n+\frac{m}{2}\right) \sin \phi \sinh r}{\sqrt{\cos ^{2} \phi+U_{0}}}\right)+\Upsilon
$$

where

$$
\begin{aligned}
\Upsilon & =\frac{d}{d z}\left(z^{-\frac{m-2}{2}} J_{\frac{m-2}{2}}(z)\right)(\xi)\left(n+\frac{m}{2}\right) \sin \phi \sinh r \\
& \times\left(\frac{1}{\sqrt{\cos ^{2} \phi+U_{0}}}-\frac{1}{\sqrt{\cos ^{2} \phi+\sinh ^{2} r+U_{\star}}}\right) \\
& \stackrel{(2.3)}{=}-\xi \cdot \xi^{-\frac{m}{2}} J_{\frac{m}{2}}(\xi)\left(n+\frac{m}{2}\right) \sin \phi \sinh r \\
& \times\left(\frac{1}{\sqrt{\cos ^{2} \phi+U_{0}}}-\frac{1}{\sqrt{\cos ^{2} \phi+\sinh ^{2} r+U_{\star}}}\right)
\end{aligned}
$$

for some $\xi \in\left(\frac{\left(n+\frac{m}{2}\right) \sin \phi \sinh r}{\sqrt{\cos ^{2} \phi+\sinh ^{2} r+U_{\star}}}, \frac{\left(n+\frac{m}{2}\right) \sin \phi \sinh r}{\sqrt{\cos ^{2} \phi+U_{0}}}\right)$.

Set

$$
\begin{aligned}
& M_{121}=\left(\cos ^{2} \phi+U_{0}\right)^{\frac{m-2}{4}} \int_{0}^{+\infty} \frac{u^{\frac{m}{2}} J_{\frac{m-2}{2}}\left(\frac{\left(n+\frac{m}{2}\right) \sin \phi}{\sqrt{\cos ^{2} \phi+U_{0}}} u\right)}{\left(\cos ^{2} \phi+U_{0}+u^{2}\right)^{\frac{1}{2}\left(n+m-\frac{1}{2}\right)}} d u \\
& M_{122}=\left(\cos ^{2} \phi+U_{0}\right)^{\frac{m-2}{4}} \int_{\sinh \zeta}^{\infty} \frac{u^{\frac{m}{2}} J_{\frac{m-2}{2}}\left(\frac{\left(n+\frac{m}{2}\right) \sin \phi}{\sqrt{\cos ^{2} \phi+U_{0}}} u\right)}{\left(\cos ^{2} \phi+U_{0}+u^{2}\right)^{\frac{1}{2}\left(n+m-\frac{1}{2}\right)}} d u
\end{aligned}
$$




$$
\begin{gathered}
E_{10}=\int_{0}^{\zeta}(\sinh r)^{\frac{m}{2}}\left(\sqrt{\frac{\sinh r}{r}}-\cosh r\right) \cdot \Theta d r, \\
E_{11}=\left(\left(n+\frac{m}{2}\right) \sin \phi\right)^{\frac{m-2}{2}} \int_{0}^{\zeta} \frac{(\sinh r)^{m-1} \cosh r}{\left(\cos ^{2} \phi+\sinh ^{2} r+U_{\star}\right)^{\frac{1}{2}\left(n+m-\frac{1}{2}\right)}} \Upsilon d r,
\end{gathered}
$$

and

$$
\begin{aligned}
E_{12}=\left(\cos ^{2} \phi+U_{0}\right)^{\frac{m-2}{4}} \int_{0}^{\zeta} \frac{(\sinh r)^{\frac{m}{2}} \cosh r J_{\frac{m-2}{2}}\left(\frac{\left(n+\frac{m}{2}\right) \sin \phi}{\sqrt{\cos ^{2} \phi+U_{0}}} \sinh r\right)}{\left(\cos ^{2} \phi+\sinh ^{2} r+U_{0}\right)^{\frac{1}{2}\left(n+m-\frac{1}{2}\right)}} \\
\quad \times\left(e^{-\frac{1}{2}\left(n+m-\frac{1}{2}\right) \ln \left[1+\frac{U_{\star}-U_{0}}{\cos ^{2} \phi+\sinh ^{2} r+U_{0}}\right]}-1\right) d r .
\end{aligned}
$$

Observe that

$$
M_{12}=\left(M_{121}-M_{122}\right)+E_{10}+E_{11}+E_{12} .
$$

We have the following (see for example [16], p. 105 or [6], p. 678):

$$
\begin{aligned}
M_{121} & =\left(\cos ^{2} \phi+U_{0}\right)^{\frac{m-2}{4}}\left(\frac{\left(n+\frac{m}{2}\right) \sin \phi}{2 \sqrt{\cos ^{2} \phi+U_{0}}}\right)^{\frac{1}{2}\left(n+m-\frac{5}{2}\right)} \\
& \times \frac{\left(\cos ^{2} \phi+U_{0}\right)^{-\frac{1}{4}\left(n-\frac{1}{2}\right)}}{\Gamma\left(\frac{1}{2}\left(n+m-\frac{1}{2}\right)\right)} K_{-\frac{1}{2}\left(n-\frac{1}{2}\right)}\left(\left(n+\frac{m}{2}\right) \sin \phi\right) \\
& =e^{-\omega^{2}}\left(\frac{\left(n+\frac{m}{2}\right) \sin \phi}{2}\right)^{\frac{1}{2}\left(n+m-\frac{5}{2}\right)} \frac{(\cos \phi)^{-\left(n-\frac{1}{2}\right)}}{\Gamma\left(\frac{1}{2}\left(n+m-\frac{1}{2}\right)\right)} \\
& \times K_{\frac{1}{2}\left(n-\frac{1}{2}\right)}\left(\left(n+\frac{m}{2}\right) \sin \phi\right)\left[1+O\left(\frac{m^{2}}{n}\right)\right]
\end{aligned}
$$

where $K_{\nu}(z)$ is modified Bessel function, and the last equality follows from the following fact:

$$
\begin{aligned}
\left(\cos ^{2} \phi+U_{0}\right)^{-\frac{1}{2}\left(n-\frac{1}{2}\right)} & =(\cos \phi)^{-\left(n-\frac{1}{2}\right)}\left(1+\frac{\omega^{2}}{n \cos \phi}\right)^{-\left(n-\frac{1}{2}\right)} \\
& =e^{-\omega^{2}}(\cos \phi)^{-\left(n-\frac{1}{2}\right)}\left[1+O\left(\frac{m^{2}}{n}\right)\right]
\end{aligned}
$$

Lemma 7.1. For $m^{3} \ll n$,

$$
M_{121} \geq \frac{1}{9} e^{-\omega^{2}}\left(\frac{1}{2}\left(n+\frac{m}{2}\right) \sin \phi\right)^{\frac{m-2}{2}} \frac{\Gamma\left(\frac{1}{2}\left(n-\frac{1}{2}\right)\right)}{\Gamma\left(\frac{1}{2}\left(n+m-\frac{1}{2}\right)\right)}
$$


Proof. Denote $\nu=\frac{1}{2}\left(n-\frac{1}{2}\right), z=\left(n+\frac{m}{2}\right) \sin \phi$. Recall that

$$
\begin{aligned}
& (\cos \phi)^{-\left(n-\frac{1}{2}\right)} K_{\frac{1}{2}\left(n-\frac{1}{2}\right)}\left(\left(n+\frac{m}{2}\right) \sin \phi\right) \\
& =\exp \left\{-\frac{\left(n-\frac{1}{2}\right)}{2} \ln \left(1-\sin ^{2} \phi\right)\right\} \int_{0}^{\infty} e^{-\frac{z}{2}\left(u+\frac{1}{u}\right)} u^{\nu-1} d u \\
& \geq \frac{1}{3} e^{\frac{n}{2} \sin ^{2} \phi} \int_{0}^{\infty} e^{-\frac{z}{2}\left(u+\frac{1}{u}\right)} u^{\nu-1} d u \\
& \geq \frac{1}{3} e^{\frac{n}{2} \sin ^{2} \phi-\frac{z^{2}}{4 \nu}} \int_{(2 \nu) / z}^{\infty} e^{-\frac{z u}{2}} u^{\nu-1} d u \\
& =\frac{1}{3} e^{-\frac{\sin ^{2} \phi}{2} O(m)}\left(\frac{2}{z}\right)^{\nu} \int_{\nu}^{\infty} e^{-u} u^{\nu-1} d u \\
& =\frac{1}{3}\left(1+O\left(\frac{m^{3}}{n}\right)\right)\left(\frac{2}{z}\right)^{\nu} \Gamma(\nu, \nu),
\end{aligned}
$$

where $\Gamma(\nu, \nu)$ is an incomplete gamma function. Recall that (see [16], p. 340, or [6], p. 901)

$$
\Gamma(\nu+1, \nu)=\nu \Gamma(\nu, \nu)+\nu^{\nu} e^{-\nu},
$$

and by the following asymptotic estimate for $\Gamma(\nu+1, \nu)$ (see (1.4) in [21]):

$$
\Gamma(\nu+1, \nu)=\Gamma(\nu+1)\left(\frac{1}{2}+\frac{1}{3}\left(\frac{2}{\nu \pi}\right)^{\frac{1}{2}}\right)+O\left(\frac{1}{\nu}\right), \nu \rightarrow \infty,
$$

it follows that

$$
\begin{aligned}
\Gamma(\nu, \nu) & \geq \nu^{-1}\left(\frac{\Gamma(\nu+1)}{2}-\nu^{\nu} e^{-\nu}\right)=\frac{\Gamma(\nu)}{2}-\nu^{\nu-1} e^{-\nu} \\
& \geq \frac{2 \Gamma(\nu)}{5}
\end{aligned}
$$

where the last inequality follows from (2.7).

Combining with (7.5), (7.6), one has, for $m^{3} \ll n$,

$$
(\cos \phi)^{-\theta} K_{\frac{1}{2} \theta}\left(\left(n+\frac{m}{2}\right) \sin \phi\right) \geq \frac{2}{15}\left(\frac{2}{\left.\left(n+\frac{m}{2}\right) \sin \phi\right)}\right)^{\frac{1}{2} \theta} \Gamma\left(\frac{1}{2} \theta\right),
$$

where $\theta=n-\frac{1}{2}$. The desired result follows easily.

Lemma 7.2. There exists some positive constant $C\left(\omega, \nu_{0}\right)$,

$$
\left|E_{10}\right|+\left|E_{11}\right|+\left|E_{12}\right|=M_{121} O\left(\frac{m}{n} \exp \left\{m^{2} C\left(\omega, \nu_{0}\right)\right\}\right) .
$$

Proof. Since the proof is similar for $E_{10}, E_{11}, E_{12}$, we only prove

$$
E_{10}=M_{121} O\left(\frac{m}{n} \exp \left\{m^{2} C\left(\omega, \nu_{0}\right)\right\}\right) .
$$

Notice that

$$
\left|\sqrt{\frac{\sinh r}{r}}-\cosh r\right| \leq c \sinh ^{2} r, \quad \forall 0<r<1
$$


(2.2) implies

$$
\begin{aligned}
\left|E_{10}\right| & \leq \frac{1}{\Gamma\left(\frac{m}{2}\right)}\left(\frac{\left(n+\frac{m}{2}\right) \sin \phi}{2}\right)^{\frac{m-2}{2}} \int_{0}^{\zeta} \frac{(\sinh r)^{m+1}}{\left(\cos ^{2} \phi+U_{0}+\sinh ^{2} r\right)^{\frac{1}{2}\left(n+m-\frac{1}{2}\right)}} d r \\
& \leq \frac{1}{\Gamma\left(\frac{m}{2}\right)}\left(\frac{\left(n+\frac{m}{2}\right) \sin \phi}{2}\right)^{\frac{m-2}{2}} \int_{0}^{\infty} \frac{(\sinh r)^{m+1}}{\left(\cos ^{2} \phi+U_{0}+\sinh ^{2} r\right)^{\frac{1}{2}\left(n+m-\frac{1}{2}\right)}} d \sinh r \\
& =\frac{1}{2 \Gamma\left(\frac{m}{2}\right)}\left(\frac{\left(n+\frac{m}{2}\right) \sin \phi}{2}\right)^{\frac{m-2}{2}}\left(\cos ^{2} \phi+U_{0}\right)^{-\frac{1}{2}\left(n-\frac{5}{2}\right)} B\left(\frac{m+2}{2}, \frac{1}{2}\left(n-\frac{5}{2}\right)\right)
\end{aligned}
$$

$$
\leq \frac{m}{4}\left(\frac{\left(n+\frac{m}{2}\right) \sin \phi}{2}\right)^{\frac{m-2}{2}}\left(\cos ^{2} \phi+U_{0}\right)^{-\frac{1}{2}\left(n-\frac{5}{2}\right)} \frac{\Gamma\left(\frac{1}{2}\left(n-\frac{5}{2}\right)\right)}{\Gamma\left(\frac{1}{2}\left(n+m-\frac{1}{2}\right)\right)} .
$$

Combining this with (17.4), (2.7) and the fact that $\sqrt{n} \phi \leq C^{*} m$, we get the desired result.

Now let us turn to

Proof of Lemma 4.3. By (2.2), one has

$$
\begin{aligned}
M_{122} & \leq \frac{1}{\Gamma\left(\frac{m}{2}\right)}\left(\frac{\left(n+\frac{m}{2}\right) \sin \phi}{2}\right)^{\frac{m-2}{2}} \int_{\sinh \zeta}^{\infty} u^{m-1}\left(\cos ^{2} \phi+U_{0}+u^{2}\right)^{-\frac{1}{2}\left(n+m-\frac{1}{2}\right)} d u \\
& \leq \frac{1}{2 \Gamma\left(\frac{m}{2}\right)}\left(\frac{\left(n+\frac{m}{2}\right) \sin \phi}{2}\right)^{\frac{m-2}{2}} \int_{\sinh \zeta}^{\infty}\left(\cos ^{2} \phi+U_{0}+u^{2}\right)^{-\frac{1}{2}\left(n+\frac{3}{2}\right)} d u^{2} \\
& =\frac{1}{\Gamma\left(\frac{m}{2}\right)}\left(\frac{\left(n+\frac{m}{2}\right) \sin \phi}{2}\right)^{\frac{m-2}{2}} \frac{1}{n-\frac{1}{2}}\left(\cos ^{2} \phi+U_{0}+\sinh ^{2} \zeta\right)^{-\frac{1}{2}\left(n-\frac{1}{2}\right)} .
\end{aligned}
$$

By (7.4) and (4.31), it is easy to see that

$$
M_{122}=M_{121} O\left(c\left(\omega, C^{*}, \nu_{o}\right) n^{-2}\right) .
$$

Combining this with (7.2), (7.9), and the above two lemmas, (4.27) holds.

Now let us proof (4.28). Obverse that

$$
\begin{aligned}
\left|M_{11}\right| & \leq c \frac{m}{\Gamma\left(\frac{m-1}{2}\right)}(\sin \phi)^{\frac{m}{2}+1} \int_{0}^{\zeta} \frac{r^{-\frac{1}{2}}(\sinh r)^{m+\frac{3}{2}}}{\left(\cos ^{2} \phi+\sinh ^{2} r+U_{\star}\right)^{\frac{1}{2}\left(n+m+\frac{3}{2}\right)}} d r \\
& \leq c \frac{m}{\Gamma\left(\frac{m-1}{2}\right)}(\sin \phi)^{\frac{m}{2}+1} \int_{0}^{+\infty} \frac{(\sinh r)^{m+1}}{\left(\cos ^{2} \phi+\sinh ^{2} r+U_{0}\right)^{\frac{1}{2}\left(n+m+\frac{3}{2}\right)}} d(\sinh r) \\
& =c \frac{m}{2 \Gamma\left(\frac{m-1}{2}\right)}(\sin \phi)^{\frac{m}{2}+1}\left(\cos ^{2} \phi+U_{0}\right)^{-\frac{1}{2}\left(n-\frac{1}{2}\right)} B\left(\frac{m}{2}+1, \frac{n}{2}-\frac{1}{4}\right) .
\end{aligned}
$$

Now

$$
B\left(\frac{m}{2}+1, \frac{n}{2}-\frac{1}{4}\right)=\frac{\Gamma\left(\frac{m}{2}+1\right) \Gamma\left(\frac{n}{2}-\frac{1}{4}\right)}{\Gamma\left(\frac{n}{2}+\frac{m}{2}+\frac{3}{4}\right)}=\frac{2 \Gamma\left(\frac{m}{2}+1\right)}{n+m-\frac{1}{2}} \frac{\Gamma\left(\frac{1}{2}\left(n-\frac{1}{2}\right)\right)}{\Gamma\left(\frac{1}{2}\left(n+m-\frac{1}{2}\right)\right)} .
$$

(7.4) implies (4.28). 


\section{ACKNOWLEDGEMENT}

This work was partially supported by the NSF of China 11171070 and 11201040 , NCET-09-0316 and "The Program for Professor of Special Appointment (Eastern Scholar) at Shanghai Institutions of Higher Learning". Part of this work was obtained in February 2009 during the first author's visit to Toulouse University. He would like to express his sincere thanks to Professor D. Bakry for the kind invitation.

\section{REFERENCES}

[1] J. M. Aldaz, The weak type $(1,1)$ bounds for the maximal function associated to cubes grow to infinity with the dimension, Ann. of Math. (2) 173 (2011), no. 2, 1013-1023, DOI 10.4007/annals.2011.173.2.10. MR2776368 (2012b:42028)

[2] Richard Beals, Bernard Gaveau, and Peter C. Greiner, Hamilton-Jacobi theory and the heat kernel on Heisenberg groups, J. Math. Pures Appl. (9) 79 (2000), no. 7, 633-689, DOI 10.1016/S0021-7824(00)00169-0. MR1776501 (2001g:35047)

[3] A. Bonfiglioli and F. Uguzzoni, Nonlinear Liouville theorems for some critical problems on Htype groups, J. Funct. Anal. 207 (2004), no. 1, 161-215, DOI 10.1016/S0022-1236(03)00138-1. MR2027639(2004k:35057)

[4] Ewa Damek, A Poisson kernel on Heisenberg type nilpotent groups, Colloq. Math. 53 (1987), no. 2, 239-247. MR924068 (89d:22006)

[5] G. B. Folland, A fundamental solution for a subelliptic operator, Bull. Amer. Math. Soc. 79 (1973), 373-376. MR0315267 (47 \#3816)

[6] I. S. Gradshteyn and I. M. Ryzhik, Table of integrals, series, and products, 7th ed., Elsevier/Academic Press, Amsterdam, 2007. Translated from the Russian. Translation edited and with a preface by Alan Jeffrey and Daniel Zwillinger. With one CD-ROM (Windows, Macintosh and UNIX). MR2360010 (2008g:00005)

[7] Yongyang Jin and Genkai Zhang, Fundamental solutions of Kohn sub-Laplacians on anisotropic Heisenberg groups and H-type groups, Canad. Math. Bull. 54 (2011), no. 1, 126140, DOI 10.4153/CMB-2010-086-1. MR2797973 (2012c:35445)

[8] Aroldo Kaplan, Fundamental solutions for a class of hypoelliptic PDE generated by composition of quadratic forms, Trans. Amer. Math. Soc. 258 (1980), no. 1, 147-153, DOI 10.2307/1998286. MR.554324 (81c:58059)

[9] Peter M. Knopf, Maximal functions on the unit n-sphere, Pacific J. Math. 129 (1987), no. 1, 77-84. MR901258(88i:42031)

[10] Hong-Quan Li, Fonctions maximales centrées de Hardy-Littlewood sur les groupes de Heisenberg, Studia Math. 191 (2009), no. 1, 89-100, DOI 10.4064/sm191-1-7 (French, with English summary). MR2471260 (2009k:42039)

[11] Hong-Quan Li, Estimations optimales du noyau de la chaleur sur les groupes de type Heisenberg, J. Reine Angew. Math. 646 (2010), 195-233, DOI 10.1515/CRELLE.2010.070 (French, with English summary). MR2719560 (2011g:58039)

[12] Hong-Quan Li, Fonctions maximales centrées de Hardy-Littlewood pour les opérateurs de Grushin. Preprint 2010.

[13] Hong-Quan Li, Remark on "Maximal functions on the unit $n$-sphere" by Peter M. Knopf (1987), Pacific J. Math. 263 (2013), no. 1, 253-256, DOI 10.2140/pjm:2013.263.253.

[14] Peter M. Knopf, Maximal functions on the unit n-sphere, Pacific J. Math. 129 (1987), no. 1, 77-84. MR.901258(88i:42031)

[15] Hong-Quan Li and Noël Lohoué, Fonction maximale centrée de Hardy-Littlewood sur les espaces hyperboliques, Ark. Mat. 50 (2012), no. 2, 359-378, DOI 10.1007/s11512-011-0163-3 (French, with French summary). MR 2961327

[16] Wilhelm Magnus, Fritz Oberhettinger, and Raj Pal Soni, Formulas and theorems for the special functions of mathematical physics, Third enlarged edition. Die Grundlehren der mathematischen Wissenschaften, Band 52, Springer-Verlag New York, Inc., New York, 1966. MR0232968 (38 \#1291) 
[17] Assaf Naor and Terence Tao, Random martingales and localization of maximal inequalities, J. Funct. Anal. 259 (2010), no. 3, 731-779, DOI 10.1016/j.jfa.2009.12.009. MR2644102 (2011k:42040)

[18] Jennifer Randall, The heat kernel for generalized Heisenberg groups, J. Geom. Anal. 6 (1996), no. 2, 287-316, DOI 10.1007/BF02921603. MR1469125(99b:22018)

[19] S. Rigot, Mass transportation in groups of type H, Commun. Contemp. Math. 7 (2005), no. 4, 509-537, DOI 10.1142/S0219199705001854. MR2166663 (2006e:49089)

[20] E. M. Stein and J.-O. Strömberg, Behavior of maximal functions in $\mathbf{R}^{n}$ for large $n$, Ark. Mat. 21 (1983), no. 2, 259-269, DOI 10.1007/BF02384314. MR727348 (86a:42027)

[21] N. M. Temme, Uniform asymptotic expansions of the incomplete gamma functions and the incomplete beta function, Math. Comp. 29 (1975), no. 132, 1109-1114. MR0387674 (52 \#8513)

[22] Kang-Hai Tan and Xiao-Ping Yang, Characterisation of the sub-Riemannian isometry groups of H-type groups, Bull. Austral. Math. Soc. 70 (2004), no. 1, 87-100, DOI 10.1017/S000497270003584X. MR2079363 (2005c:53033)

[23] N. Th. Varopoulos, L. Saloff-Coste, and T. Coulhon, Analysis and geometry on groups, Cambridge Tracts in Mathematics, vol. 100, Cambridge University Press, Cambridge, 1992. MR $1218884(95 \mathrm{f}: 43008)$

[24] G. N. Watson, A Treatise on the Theory of Bessel Functions, Cambridge University Press, Cambridge, England, 1944. MR0010746 (6,64a)

[25] J. M. Zhao, N. Q. Song, Maximal function on the quaternion Heisenberg groups. Preprint 2010 .

School of Mathematical Sciences, Fudan University, 220 Handan Road, Shanghai 200433, People's Republic of China

E-mail address: hongquan_li@fudan.edu.cn

E-mail address: hong-quanli@yahoo.fr

School of Mathematics and Statistics, Changshu Institute of Technology 215500 , Changshu, People's Republic of China

E-mail address: binqiancn@yahoo.com.cn

E-mail address: binqiancn@gmail.com 